Acta Crystallographica Section E

Structure Reports

Online

ISSN 1600-5368

Editors: W. Clegg and D. G. Watson

\title{
Dipotassium biphenyl-4,4'-disulfonate dihydrate: a coordination polymer
}

\author{
Graham Smith, Urs D. Wermuth and Peter C. Healy
}

Copyright $($ International Union of Crystallography

Author(s) of this paper may load this reprint on their own web site or institutional repository provided that this cover page is retained. Republication of this article or its storage in electronic databases other than as specified above is not permitted without prior permission in writing from the IUCr.

For further information see http://journals.iucr.org/services/authorrights.html 
Acta Crystallographica Section E

\section{Structure Reports}

Online

ISSN 1600-5368

\section{Dipotassium biphenyl-4, $\mathbf{4}^{\prime}$-disulfonate dihydrate: a coordination polymer}

\author{
Graham Smith, $^{\mathrm{a} *}$ Urs D. Wermuth ${ }^{\mathrm{a}}$ and Peter C. Healy ${ }^{\mathrm{b}}$

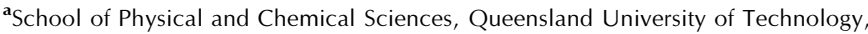 \\ GPO Box 2434, Brisbane, Queensland 4001, Australia, and ${ }^{\mathbf{b}}$ School of Biomolecular \\ and Physical Sciences, Griffith University, Nathan, Queensland 4111, Australia \\ Correspondence e-mail: g.smith@qut.edu.au
}

Received 29 October 2007; accepted 7 November 2007

Key indicators: single-crystal X-ray study; $T=297 \mathrm{~K}$; mean $\sigma(\mathrm{C}-\mathrm{C})=0.006 \AA$; $R$ factor $=0.031 ; w R$ factor $=0.083 ;$ data-to-parameter ratio $=10.1$.

The polymeric structure of the title compound, poly[[diaquabis $(\mu$-biphenyl-4,4'-disulfonato)tetrapotassium(I)] dihydrate], $\left\{\left[\mathrm{K}_{2}\left(\mathrm{C}_{12} \mathrm{H}_{8} \mathrm{O}_{6} \mathrm{~S}_{2}\right)\left(\mathrm{H}_{2} \mathrm{O}\right)\right] \cdot \mathrm{H}_{2} \mathrm{O}\right\}_{n}$, is based on an asymmetric unit comprising three independent and different potassium centres, one six-coordinate $[\mathrm{K}-\mathrm{O}=2.657$ (3)$2.866(5) \AA]$, one seven-coordinate $[\mathrm{K}-\mathrm{O}=2.703(3)-$ $3.040(4) \AA]$, and the third ten-coordinate $[\mathrm{K}-\mathrm{O}=$ $2.751(3)-3.079$ (4) $\AA$ ], with two of these lying on crystallographic mirror planes. The four half-occupancy water molecules also lie on the mirror planes with two coordinated (one monodentate, the other bidentate bridging) and two as molecules of solvation. The interlinked coordination polyhedra form chains which are joined laterally through the biphenyl residues as well as through head-to-tail water hydrogen-bonding interactions, giving a two-dimensional structure.

\section{Related literature}

For other 4,4'-biphenyldisulfonate crystal structures, see: Swift et al. (1998); Liao et al. (2001); Cai et al. (2001); Usuki et al. (2002). For related literature, see: Pivovar et al. (2002). For synthesis, see: Feldmann (1931).

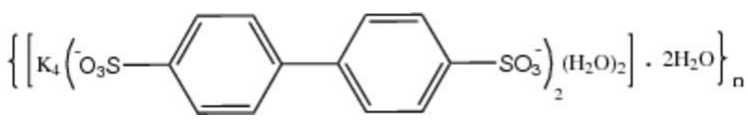

\section{Experimental}

Crystal data

$\left[\mathrm{K}_{2}\left(\mathrm{C}_{12} \mathrm{H}_{8} \mathrm{O}_{6} \mathrm{~S}_{2}\right)\left(\mathrm{H}_{2} \mathrm{O}\right)\right] \cdot \mathrm{H}_{2} \mathrm{O}$

$M_{r}=426.56$

Monoclinic, $\mathrm{Cm}$

$a=5.8316(10) \AA$

$b=19.691(7) \AA$

$c=14.623(2) \AA$

$\beta=98.953(13)$

\author{
Data collection \\ Rigaku AFC 7R diffractometer \\ Absorption correction: $\psi$ scan \\ (TEXSAN for Windows; Mole- \\ cular Structure Corporation, \\ 1999) \\ $T_{\min }=0.774, T_{\max }=0.849$ \\ 2295 independent reflections \\ 2196 reflections with $I>2 \sigma(I)$ \\ $R_{\text {int }}=0.016$ \\ 3 standard reflections \\ frequency: $150 \mathrm{~min}$ \\ intensity decay: $0.6 \%$
}

2295 measured reflections

Refinement

$R\left[F^{2}>2 \sigma\left(F^{2}\right)\right]=0.031$

$w R\left(F^{2}\right)=0.083$

$S=0.88$

2295 reflections

227 parameters

2 restraints

\section{Table 1}

Selected bond lengths $(\AA)$.

\begin{tabular}{llll}
\hline $\mathrm{K} 1-\mathrm{O} 43 B$ & $2.850(4)$ & $\mathrm{K} 2-\mathrm{O} 42 A^{\text {vii }}$ & $2.741(3)$ \\
$\mathrm{K} 1-\mathrm{O} 41 B^{\mathrm{i}}$ & $2.703(3)$ & $\mathrm{K} 3-\mathrm{O} 2 W$ & $3.079(4)$ \\
$\mathrm{K} 1-\mathrm{O} 41 A^{\text {ii }}$ & $3.040(4)$ & $\mathrm{K} 3-\mathrm{O} 41 B$ & $2.751(3)$ \\
$\mathrm{K} 1-\mathrm{O} 43 A^{\text {ii }}$ & $3.058(3)$ & $\mathrm{K} 3-\mathrm{O} 42 B$ & $3.076(4)$ \\
$\mathrm{K} 1-\mathrm{O} 42 A^{\text {iii }}$ & $2.866(3)$ & $\mathrm{K} 3-\mathrm{O} 42 A^{\text {iii }}$ & $2.938(3)$ \\
$\mathrm{K} 1-\mathrm{O} 43 B^{\text {iv }}$ & $2.739(3)$ & $\mathrm{K} 3-\mathrm{O} 43 A^{\text {iii }}$ & $3.027(3)$ \\
$\mathrm{K} 1-\mathrm{O} 41 A^{\mathrm{v}}$ & $2.681(3)$ & $\mathrm{K} 3-\mathrm{O} 2 W^{\text {viii }}$ & $2.793(4)$ \\
$\mathrm{K} 2-\mathrm{O} 1 W$ & $2.703(6)$ & $\mathrm{K} 3-\mathrm{O} 41 B^{\text {ix }}$ & $2.751(3)$ \\
$\mathrm{K} 2-\mathrm{O} 2 W$ & $2.866(5)$ & $\mathrm{K} 3-\mathrm{O} 42 B^{\text {ix }}$ & $3.076(4)$ \\
$\mathrm{K} 2-\mathrm{O} 43 A^{\text {ii }}$ & $2.657(3)$ & $\mathrm{K} 3-\mathrm{O} 42 A^{\text {vii }}$ & $2.938(3)$ \\
$\mathrm{K} 2-\mathrm{O} 42 A^{\text {iii }}$ & $2.741(3)$ & $\mathrm{K} 3-\mathrm{O} 43 A^{\text {vii }}$ & $3.027(3)$ \\
$\mathrm{K} 2-\mathrm{O} 43 A^{\text {vi }}$ & $2.657(3)$ & &
\end{tabular}

Symmetry codes: (i) $x-1, y, z$; (ii) $x-1, y, z+1$; (iii) $x, y, z+1$; (iv) $x-\frac{1}{2},-y+\frac{1}{2}, z$; (v) $x-\frac{1}{2},-y+\frac{1}{2}, z+1$; (vi) $x-1,-y, z+1$; (vii) $x,-y, z+1$; (viii) $x+1, y, z$; (ix) $x,-y, z$.

Table 2

Hydrogen-bond geometry $\left(\AA{ }^{\circ}\right)$.

\begin{tabular}{|c|c|c|c|c|}
\hline$D-\mathrm{H} \cdots A$ & $D-\mathrm{H}$ & $\mathrm{H} \cdots A$ & $D \cdots A$ & $D-\mathrm{H} \cdots A$ \\
\hline $\mathrm{O} 1 W-\mathrm{H} 11 W \cdots \mathrm{O} 4 W^{\mathrm{iii}}$ & 0.97 & 2.18 & $2.778(9)$ & 119 \\
\hline $\mathrm{O} 2 W-\mathrm{H} 2 W \cdots \mathrm{O} 42 B$ & 0.96 & 1.91 & $2.840(5)$ & 162 \\
\hline $\mathrm{O} 3 W-\mathrm{H} 3 W \cdots \mathrm{O} 42 B$ & 0.96 & 1.95 & $2.884(5)$ & 162 \\
\hline $\mathrm{O} 4 W-\mathrm{H} 41 W \cdots \mathrm{O} 3 W$ & 0.90 & 1.82 & $2.715(9)$ & 180 \\
\hline $\mathrm{O} 4 W-\mathrm{H} 41 W \cdots \mathrm{O} 3 W$ & 0.90 & 1.82 & $2.715(9)$ & 180 \\
\hline $\mathrm{C} 5 A-\mathrm{H} 5 A \cdots \mathrm{O} 43 A$ & 0.95 & 2.50 & $2.916(5)$ & 106 \\
\hline $\mathrm{C} 5 B-\mathrm{H} 5 B \cdots \mathrm{O} 41 B$ & 0.94 & 2.47 & $2.873(5)$ & 106 \\
\hline
\end{tabular}

Symmetry code: (iii) $x, y, z+1$.

Data collection: MSC/AFC Diffractometer Control Software (Molecular Structure Corporation, 1999); cell refinement: $M S C / A F C$ Diffractometer Control Software; data reduction: TEXSAN for Windows (Molecular Structure Corporation, 1999); program(s) used to solve structure: SIR92 (Altomare et al., 1994); program(s) used to refine structure: SHELXL97 (Sheldrick, 1997); molecular graphics: PLATON (Spek, 2003); software used to prepare material for publication: PLATON.

The authors acknowledge financial support from the School of Physical and Chemical Sciences, Queensland University of Technology, and the School of Biomolecular and Physical Sciences, Griffith University. 


\section{metal-organic compounds}

Supplementary data and figures for this paper are available from the IUCr electronic archives (Reference: TK2210).

\section{References}

Altomare, A., Cascarano, G., Giacovazzo, C., Guagliardi, A., Burla, M. C., Polidori, G. \& Camalli, M. (1994). J. Appl. Cryst. 27, 435.

Cai, J., Hu, X.-P., Yao, J.-H. \& Ji, L.-N. (2001). Inorg. Chem. Commun. 4, 478482.

Feldmann, J. (1931). Helv. Chim. Acta, 14, 751-778.

Flack, H. D. (1983). Acta Cryst. A39, 876-881.
Liao, C.-Z., Feng, X.-L., Yao, J.-H. \& Cai, J.-W. (2001). Acta Cryst. C57, 12151216.

Molecular Structure Corporation (1999). MSC/AFC Diffractometer Control Software and TEXSAN for Windows (Version 1.06). MSC, The Woodlands, Texas, USA.

Pivovar, A. M., Ward, M. D., Brown, C. M. \& Neumann, D. A. (2002). J. Phys. Chem. 106, 4916-4924.

Sheldrick, G. M. (1997). SHELXL97. University of Göttingen, Germany.

Spek, A. L. (2003). J. Appl. Cryst. 36, 7-13.

Swift, J. A., Pivovar, A. M., Reynolds, A. M. \& Ward, M. D. (1998). J. Am. Chem. Soc. 120, 5887-5894.

Usuki, N., Ohba, M. \& Okawa, H. (2002). J. Chem. Soc. Jpn, 75, 16931698. 


\section{supplementary materials}




\section{supplementary materials}

Acta Cryst. (2007). E63, m3056-m3057 [ doi:10.1107/S1600536807056930 ]

\section{Dipotassium biphenyl-4,4'-disulfonate dihydrate: a coordination polymer}

\section{G. Smith, U. D. Wermuth and P. C. Healy}

\section{Comment}

Compounds of 4,4'-biphenyldisulfonic acid (BPDS) are not numerous in the crystallographic literature. The guanidinium salts have been used for the generation of 2-D structures for the formation of crystalline clathrates with aromatic hydrocarbons (Swift et al., 1998; Pivovar et al., 2002). The bis(alaninium) salt is also known (Liao et al., 2001). With coordination compounds, BDPDS is generally found as a dianionic counter ion (Cai et al., 2001; Usuki et al., 2002). We obtained X-ray diffraction quality crystals of the hydrated dipotassium salt of BPDS as an intermediate in the synthesis of BPDS, when recrystallized from water. The structure of this compound, $\mathrm{K}_{2}{ }^{2+} \cdot \mathrm{C}_{12} \mathrm{H}_{8} \mathrm{O}_{6} \mathrm{~S}_{2}{ }^{2-} \cdot 2 \mathrm{H}_{2} \mathrm{O}$ (I) is reported here.

The structure of (I) is based on an asymmetric unit comprising three independent and different potassium centres, one six-coordinate (K2) [K-O range, 2.657 (3)-2.866 (5) $\AA$ ], one seven- coordinate (K1) [K-O range, 2.703 (3)-3.040 (4) $\AA$ ] and the third ten-coordinate (K3) [K-O range, 2.751 (3)-3.079 (4) $\AA$ ], with two of these (K2 and K3) lying on crystallographic mirror planes (Fig. 1). The four half-occupancy water molecules also lie on the mirror planes with two coordinated [one monodentate (O1W on K2), and one bidentate (O2W, bridging $\mathrm{K} 2$ and $\mathrm{K} 3)$ ], and the other two (OW3, OW4) as molecules of solvation. The structure has pseudo $2 / \mathrm{m}$ symmetry, the 2-fold rotational symmetry along the $b$ axis being upset largely by the differing roles of the water molecules in the structure. This was also consistent with the failure to obtain a solution of the structure in the space group $C 2 / m$.

The interlinked potassium coordination polyhedra form chains which extend down the $b$ axix and are linked laterally across the $c$ cell direction through the biphenyl residues of the BPDS ligands, giving a 2-D structure (Fig. 2). This is somewhat analogous to the 2-D but hydrogen-bonded guanidinium-BPDS open framework structures (Swift et al., 1998; Pivovar et al., 2002) which accommodate interstitial inert aromatic molecules. With (I), the water molecules are similarly accommodated in the interstitial spaces along the crystallographic mirror planes in linear head-to-tail interactions (Table 1) and also link the coordination polymer chains. In addition, the coordinated water molecules give lateral $O-H \cdots \mathrm{O}_{\text {sulfonate }}$ interactions within the polymer chains.

Within the BPDS anion, the two phenyl rings (A and B) are close to co-planar [torsion angle $\mathrm{C} 2 \mathrm{~A}-\mathrm{C} 1 \mathrm{~A}-\mathrm{C} 1 \mathrm{~B}-\mathrm{C} 6 \mathrm{~B}$, $-178.3(5)^{\circ}$ ], which is similar to that found in the alaninium salt where the two residues are inversion related (Liao et al., 2001).

\section{Experimental}

The title compound was obtained as colourless crystals from the room temperature evaporation of an aqueous solution of dipotassium 4,4-biphenyldisulfonate, an intermediate product in the synthesis of 4,4'-biphenyldisulfonic acid by the sulfonation of biphenyl using the procedure of Feldmann (1931). 


\section{supplementary materials}

\section{Refinement}

Hydrogen atoms on the water molecules were located by difference methods but their positional and isotropic displacement parameters were fixed as located and $U_{\text {iso }}(\mathrm{H})=1.2 U_{\text {eq }}(\mathrm{O})$, respectively; see Table 1 for distances. The aromatic $\mathrm{H}$ atoms were included in the refinement in their calculated positions $(\mathrm{C}-\mathrm{H}=0.94-0.95 \AA)$ using a riding model approximation, with $U_{\text {iso }}(\mathrm{H})=1.2 U_{\text {eq }}(\mathrm{C})$.

\section{Figures}

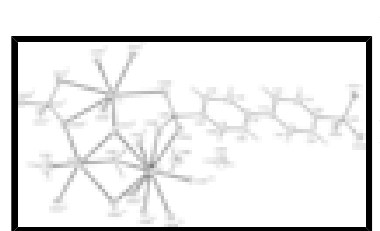

Fig. 1. Molecular configuration and atom naming scheme for the BPDS ligand, the three independent potassium coordination polyhedra and the water molecules in the asymmetric unit of (I). Displacement ellipsoids are drawn at the 50\% probability level. Dashed lines indicate hydrogen-bonding interactions associated with the water molecules. The two potassium ions $(\mathrm{K} 2, \mathrm{~K} 3)$ and the four water molecules $(\mathrm{O} 1 \mathrm{~W}-\mathrm{O} 4 \mathrm{~W})$ lie on a crystallographic mirror plane. Symmetry codes: (i) $x-1, y, z$; (ii) $x-1, y, z+1$; (iii) $x, y, z+1$; (iv) $x-1 / 2,-y+1 / 2, z$; (v) $x-1 / 2,-y+1 / 2, z+1$; (vi) $x-1,-y, z+1$; (vii) $x,-y, z+1$; (viii) $x+1, y, z$; (ix) $x,-y, z$.

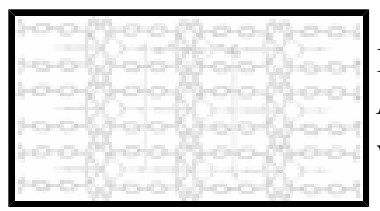

Fig. 2. The 2-D hydrogen-bonded structure of (I) viewed down the $a$ axis, showing $K-\mathrm{O}_{\text {sulfonate }}$ coordination-polymer chains with the biphenyl step linkages, together with the water mediated hydrogen-bonding associations lying on the mirror planes at $y=0,1 / 2$.

\section{dipotassium biphenyl-4,4'-disulfonate dihydrate}

$$
\begin{aligned}
& \text { Crystal data } \\
& 2 \mathrm{~K}^{+} \cdot \mathrm{C}_{12} \mathrm{H}_{8} \mathrm{O}_{6} \mathrm{~S}_{2}{ }^{2-} \cdot 2 \mathrm{H}_{2} \mathrm{O} \\
& M_{r}=426.56 \\
& \text { Monoclinic, } C m \\
& \text { Hall symbol: } \mathrm{C}-2 \mathrm{y} \\
& a=5.8316(10) \AA \\
& b=19.691(7) \AA \\
& c=14.623(2) \AA \\
& \beta=98.953(13)^{\circ} \\
& V=1658.7(7) \AA^{3} \\
& Z=4
\end{aligned}
$$

\section{Data collection}

Rigaku AFC 7R

diffractometer

Radiation source: rotating anode

Monochromator: graphite

$T=297(2) \mathrm{K}$

$\omega-2 \theta$ scans

Absorption correction: $\psi$ scan

$$
\begin{aligned}
& F_{000}=872 \\
& D_{\mathrm{x}}=1.708 \mathrm{Mg} \mathrm{m}^{-3} \\
& \text { Mo } K \alpha \text { radiation } \\
& \lambda=0.71073 \AA \\
& \text { Cell parameters from } 25 \text { reflections } \\
& \theta=12.9-17.1^{\circ} \\
& \mu=0.86 \mathrm{~mm}^{-1} \\
& T=297(2) \mathrm{K} \\
& \text { Block, colourless } \\
& 0.32 \times 0.25 \times 0.20 \mathrm{~mm}
\end{aligned}
$$

$R_{\text {int }}=0.016$

$\theta_{\max }=27.5^{\circ}$

$\theta_{\text {min }}=2.5^{\circ}$

$h=-3 \rightarrow 7$

$k=0 \rightarrow 25$

$l=-18 \rightarrow 18$ 


\section{supplementary materials}

(TEXSAN for Windows; Molecular Structure Corporation, 1999)

$T_{\min }=0.774, T_{\max }=0.849$

3 standard reflections

2295 measured reflections every $150 \mathrm{~min}$

2295 independent reflections

intensity decay: $0.6 \%$

2196 reflections with $I>2 \sigma(I)$

\section{Refinement}

Refinement on $F^{2}$

Least-squares matrix: full

$R\left[F^{2}>2 \sigma\left(F^{2}\right)\right]=0.031$

$w R\left(F^{2}\right)=0.083$

$S=0.88$

2295 reflections

227 parameters

2 restraints

Hydrogen site location: inferred from neighbouring sites

$\mathrm{H}$-atom parameters not refined

$$
w=1 /\left[\sigma^{2}\left(F_{\mathrm{o}}{ }^{2}\right)+(0.1 P)^{2}+15.7726 P\right]
$$

where $P=\left(F_{\mathrm{o}}{ }^{2}+2 F_{\mathrm{c}}{ }^{2}\right) / 3$

$(\Delta / \sigma)_{\max }=0.002$

$\Delta \rho_{\max }=0.40 \mathrm{e} \AA^{-3}$

$\Delta \rho_{\min }=-0.39$ e $\AA^{-3}$

Extinction correction: none

Absolute structure: Flack (1983)

Primary atom site location: structure-invariant direct methods

Flack parameter: $0.02(8)$

Secondary atom site location: difference Fourier map

\section{Special details}

Geometry. Bond distances, angles etc. have been calculated using the rounded fractional coordinates. All su's are estimated from the variances of the (full) variance-covariance matrix. The cell e.s.d.'s are taken into account in the estimation of distances, angles and torsion angles

Refinement. Refinement of $\mathrm{F}^{2}$ against ALL reflections. The weighted $R$-factor $w R$ and goodness of fit $\mathrm{S}$ are based on $\mathrm{F}^{2}$, conventional $R$-factors $R$ are based on $\mathrm{F}$, with $\mathrm{F}$ set to zero for negative $\mathrm{F}^{2}$. The threshold expression of $\mathrm{F}^{2}>2$ sigma( $\left.\mathrm{F}^{2}\right)$ is used only for calculating $R$-factors (gt) etc. and is not relevant to the choice of reflections for refinement. $R$-factors based on $\mathrm{F}^{2}$ are statistically about twice as large as those based on F, and R- factors based on ALL data will be even larger.

Fractional atomic coordinates and isotropic or equivalent isotropic displacement parameters $\left(\AA^{2}\right)$

$\begin{array}{lllll} & x & y & z & U_{\text {iso }} * U_{\text {eq }} \\ \mathrm{K} 1 & 0.27341(18) & 0.18494(4) & 0.50427(7) & 0.0306(2) \\ \mathrm{K} 2 & 0.3806(2) & 0.00000 & 0.66340(8) & 0.0322(3) \\ \mathrm{K} 3 & 0.8140(2) & 0.00000 & 0.48912(8) & 0.0331(3) \\ \mathrm{S} 4 \mathrm{~A} & 0.85074(12) & 0.13645(4) & -0.35496(5) & 0.0216(2) \\ \mathrm{S} 4 \mathrm{~B} & 0.67991(14) & 0.14568(5) & 0.35851(6) & 0.0255(2) \\ \text { O1W } & 0.4854(11) & 0.00000 & 0.8501(4) & 0.080(3) \\ \text { O2W } & 0.2800(7) & 0.00000 & 0.4652(3) & 0.0354(12) \\ \text { O41A } & 0.8714(6) & 0.20502(15) & -0.3888(2) & 0.0431(9) \\ \text { O41B } & 0.9000(5) & 0.12170(17) & 0.40854(18) & 0.0370(9) \\ \text { O42A } & 0.6357(5) & 0.10358(17) & -0.39554(19) & 0.0374(9) \\ \text { O42B } & 0.4950(6) & 0.09558(17) & 0.3603(2) & 0.0404(10)\end{array}$




\section{supplementary materials}

$\begin{array}{lllll}\text { O43A } & 1.0527(5) & 0.09479(17) & -0.3630(2) & 0.0403(10) \\ \text { O43B } & 0.6088(6) & 0.21186(17) & 0.3865(2) & 0.0389(9) \\ \text { C1A } & 0.7952(7) & 0.15078(19) & -0.0464(3) & 0.0274(10) \\ \text { C1B } & 0.7699(7) & 0.1525(2) & 0.0539(3) & 0.0285(10) \\ \text { C2A } & 0.6400(8) & 0.1846(3) & -0.1132(3) & 0.0459(15) \\ \text { C2B } & 0.5868(9) & 0.1849(2) & 0.0848(3) & 0.0427(14) \\ \text { C3A } & 0.6591(9) & 0.1809(2) & -0.2069(3) & 0.0424(15) \\ \text { C3B } & 0.5603(8) & 0.1853(2) & 0.1778(3) & 0.0429(15) \\ \text { C4A } & 0.8342(7) & 0.14383(19) & -0.2346(2) & 0.0253(10) \\ \text { C4B } & 0.7182(7) & 0.1511(2) & 0.2407(3) & 0.0273(10) \\ \text { C5A } & 0.9927(8) & 0.1114(3) & -0.1700(3) & 0.0497(16) \\ \text { C5B } & 0.9036(10) & 0.1196(4) & 0.2123(3) & 0.065(2) \\ \text { C6A } & 0.9716(9) & 0.1150(3) & -0.0765(3) & 0.0533(18) \\ \text { C6B } & 0.9281(9) & 0.1203(4) & 0.1193(3) & 0.066(2) \\ \text { O3W } & 0.3491(11) & 0.00000 & 0.2133(3) & 0.0608(19) \\ \text { O4W } & 0.4275(14) & 0.00000 & 0.0351(5) & 0.083(2) \\ \text { H2A } & 0.51870 & 0.20980 & -0.09300 & 0.0550^{*} \\ \text { H2B } & 0.47780 & 0.20740 & 0.04090 & 0.0510^{*} \\ \text { H2W } & 0.34800 & 0.03900 & 0.44000 & 0.0420^{*} \\ \text { H3A } & 0.55160 & 0.20360 & -0.25180 & 0.0500^{*} \\ \text { H3B } & 0.43380 & 0.20770 & 0.19790 & 0.0510^{*} \\ \text { H5A } & 1.11400 & 0.08620 & -0.19020 & 0.0600^{*} \\ \text { H5B } & 1.01260 & 0.09710 & 0.25620 & 0.0780^{*} \\ \text { H6A } & 0.09230 & -0.03160 & 0.0640^{*} \\ \text { H6B } & 0.09790 & 0.09920 & 0.0790^{*} \\ \text { H11W } & 0.000000 .0960^{*} \\ \text { H12W } & 0.000000 & 0.88400 & 0.0960^{*} \\ \text { H3W } & 0.03850 & 0.88400 & 0.0730^{*} \\ \text { H41W } & 0.00000 & 0.25200 & 0.0970^{*} \\ \text { H42W } & 0.00000 & 0.09400 & 0.0970^{*}\end{array}$

Atomic displacement parameters $\left(\AA^{2}\right)$

$\begin{array}{lllllll} & U^{11} & U^{22} & U^{33} & U^{12} & U^{13} & U^{23} \\ \mathrm{~K} 1 & 0.0334(4) & 0.0286(4) & 0.0307(3) & -0.0007(4) & 0.0082(3) & 0.0001(4) \\ \mathrm{K} 2 & 0.0286(6) & 0.0346(6) & 0.0344(6) & 0.0000 & 0.0083(5) & 0.0000 \\ \mathrm{~K} 3 & 0.0357(6) & 0.0317(5) & 0.0325(6) & 0.0000 & 0.0076(5) & 0.0000 \\ \mathrm{~S} 4 \mathrm{~A} & 0.0243(4) & 0.0239(4) & 0.0174(4) & 0.0011(3) & 0.0058(3) & 0.0000(3) \\ \text { S4B } & 0.0310(4) & 0.0297(4) & 0.0166(4) & -0.0042(4) & 0.0062(3) & -0.0006(3) \\ \text { O1W } & 0.073(4) & 0.116(6) & 0.045(3) & 0.0000 & -0.005(3) & 0.0000 \\ \text { O2W } & 0.038(2) & 0.036(2) & 0.035(2) & 0.0000 & 0.0147(17) & 0.0000 \\ \text { O41A } & 0.076(2) & 0.0276(14) & 0.0285(14) & -0.0031(16) & 0.0174(15) & 0.0044(11) \\ \text { O41B } & 0.0383(16) & 0.0489(17) & 0.0230(13) & 0.0000(14) & 0.0020(11) & 0.0051(12) \\ \text { O42A } & 0.0328(14) & 0.0533(18) & 0.0267(14) & -0.0133(14) & 0.0063(11) & -0.0078(12) \\ \text { O42B } & 0.0453(17) & 0.0468(19) & 0.0302(14) & -0.0183(15) & 0.0095(13) & 0.0004(13) \\ \text { O43A } & 0.0370(16) & 0.057(2) & 0.0279(14) & 0.0217(15) & 0.0085(12) & 0.0012(13) \\ \text { O43B } & 0.0518(17) & 0.0391(16) & 0.0277(14) & 0.0023(14) & 0.0120(13) & -0.0056(12) \\ \text { C1A } & 0.0342(19) & 0.0320(18) & 0.0168(15) & 0.0031(16) & 0.0068(14) & 0.0009(13)\end{array}$


supplementary materials

$\begin{array}{lllllll}\text { C1B } & & & & & \\ \text { C2A } & 0.0317(19) & 0.0359(19) & 0.0181(16) & 0.0004(16) & 0.0041(14) & -0.0003(14) \\ \text { C2B } & 0.051(3) & 0.069(3) & 0.0196(17) & 0.030(2) & 0.0114(18) & 0.0024(18) \\ \text { C3A } & 0.049(2) & 0.058(3) & 0.0222(19) & 0.024(2) & 0.0086(17) & 0.0073(19) \\ \text { C3B } & 0.049(3) & 0.059(3) & 0.0196(18) & 0.025(2) & 0.0071(17) & 0.0072(17) \\ \text { C4A } & 0.050(3) & 0.056(3) & 0.0252(19) & 0.025(2) & 0.0140(18) & 0.0019(17) \\ \text { C4B } & 0.0306(18) & 0.0305(17) & 0.0154(15) & -0.0001(15) & 0.0057(14) & -0.0018(13) \\ \text { C5A } & 0.0318(19) & 0.0332(19) & 0.0170(16) & -0.0034(15) & 0.0041(14) & -0.0016(13) \\ \text { C5B } & 0.044(2) & 0.084(4) & 0.0220(18) & 0.036(3) & 0.0079(17) \\ \text { C6A } & 0.061(3) & 0.116(5) & 0.0188(19) & 0.048(3) & 0.001(2) \\ \text { C6B } & 0.052(3) & 0.085(4) & 0.0229(18) & 0.037(3) & 0.0059(18) \\ \text { O3W } & 0.054(3) & 0.122(5) & 0.023(2) & 0.053(3) & 0.014(2) & 0.006(2) \\ \text { O4W } & 0.080(4) & 0.057(3) & 0.040(3) & 0.0000 & 0.016(3) & -0.008(3)\end{array}$

Geometric parameters $\left(A,{ }^{\circ}\right)$

\begin{tabular}{|c|c|c|c|}
\hline $\mathrm{K} 1-\mathrm{O} 43 \mathrm{~B}$ & $2.850(4)$ & $\mathrm{S} 4 \mathrm{~B}-\mathrm{C} 4 \mathrm{~B}$ & $1.775(4)$ \\
\hline $\mathrm{K} 1-\mathrm{O} 41 \mathrm{~B}^{\mathrm{i}}$ & $2.703(3)$ & $\mathrm{O} 1 \mathrm{~W}-\mathrm{H} 12 \mathrm{~W}$ & 0.8300 \\
\hline $\mathrm{K} 1-\mathrm{O} 41 \mathrm{~A}^{\mathrm{ii}}$ & $3.040(4)$ & $\mathrm{O} 1 \mathrm{~W}-\mathrm{H} 11 \mathrm{~W}$ & 0.9700 \\
\hline $\mathrm{K} 1-\mathrm{O} 43 \mathrm{~A}^{\mathrm{ii}}$ & $3.058(3)$ & $\mathrm{O} 2 \mathrm{~W}-\mathrm{H} 2 \mathrm{~W}^{\mathrm{ix}}$ & 0.9600 \\
\hline $\mathrm{K} 1-\mathrm{O} 42 \mathrm{~A}^{\mathrm{iii}}$ & $2.866(3)$ & $\mathrm{O} 2 \mathrm{~W}-\mathrm{H} 2 \mathrm{~W}$ & 0.9600 \\
\hline $\mathrm{K} 1-\mathrm{O} 43 \mathrm{~B}^{\mathrm{iv}}$ & $2.739(3)$ & $\mathrm{O} 3 \mathrm{~W}-\mathrm{H} 3 \mathrm{~W}$ & 0.9600 \\
\hline $\mathrm{K} 1-\mathrm{O} 41 \mathrm{~A}^{\mathrm{v}}$ & $2.681(3)$ & $\mathrm{O} 3 \mathrm{~W}-\mathrm{H} 3 \mathrm{~W}^{\mathrm{ix}}$ & 0.9600 \\
\hline $\mathrm{K} 2-\mathrm{O} 1 \mathrm{~W}$ & $2.703(6)$ & $\mathrm{O} 4 \mathrm{~W}-\mathrm{H} 41 \mathrm{~W}$ & 0.9000 \\
\hline $\mathrm{K} 2-\mathrm{O} 2 \mathrm{~W}$ & $2.866(5)$ & $\mathrm{O} 4 \mathrm{~W}-\mathrm{H} 42 \mathrm{~W}$ & 0.8800 \\
\hline $\mathrm{K} 2-\mathrm{O} 43 \mathrm{~A}^{\mathrm{ii}}$ & $2.657(3)$ & $\mathrm{C} 1 \mathrm{~A}-\mathrm{C} 6 \mathrm{~A}$ & $1.375(7)$ \\
\hline $\mathrm{K} 2-\mathrm{O} 42 \mathrm{~A}^{\mathrm{iii}}$ & $2.741(3)$ & $\mathrm{C} 1 \mathrm{~A}-\mathrm{C} 2 \mathrm{~A}$ & $1.393(6)$ \\
\hline $\mathrm{K} 2-\mathrm{O} 43 \mathrm{~A}^{\mathrm{vi}}$ & $2.657(3)$ & $\mathrm{C} 1 \mathrm{~A}-\mathrm{C} 1 \mathrm{~B}$ & $1.497(6)$ \\
\hline $\mathrm{K} 2-\mathrm{O} 42 \mathrm{~A}^{\mathrm{vii}}$ & $2.741(3)$ & $\mathrm{C} 1 \mathrm{~B}-\mathrm{C} 2 \mathrm{~B}$ & $1.379(6)$ \\
\hline $\mathrm{K} 3-\mathrm{O} 2 \mathrm{~W}$ & $3.079(4)$ & $\mathrm{C} 1 \mathrm{~B}-\mathrm{C} 6 \mathrm{~B}$ & $1.376(7)$ \\
\hline $\mathrm{K} 3-\mathrm{O} 41 \mathrm{~B}$ & $2.751(3)$ & $\mathrm{C} 2 \mathrm{~A}-\mathrm{C} 3 \mathrm{~A}$ & $1.394(6)$ \\
\hline $\mathrm{K} 3-\mathrm{O} 42 \mathrm{~B}$ & $3.076(4)$ & $\mathrm{C} 2 \mathrm{~B}-\mathrm{C} 3 \mathrm{~B}$ & $1.392(6)$ \\
\hline $\mathrm{K} 3-\mathrm{O} 42 \mathrm{~A}^{\mathrm{iii}}$ & $2.938(3)$ & $\mathrm{C} 3 \mathrm{~A}-\mathrm{C} 4 \mathrm{~A}$ & $1.367(6)$ \\
\hline $\mathrm{K} 3-\mathrm{O} 43 \mathrm{~A}^{\mathrm{iii}}$ & $3.027(3)$ & $\mathrm{C} 3 \mathrm{~B}-\mathrm{C} 4 \mathrm{~B}$ & $1.373(6)$ \\
\hline $\mathrm{K} 3-\mathrm{O} 2 \mathrm{~W}^{\mathrm{viii}}$ & $2.793(4)$ & $\mathrm{C} 4 \mathrm{~A}-\mathrm{C} 5 \mathrm{~A}$ & $1.372(6)$ \\
\hline $\mathrm{K} 3-\mathrm{O} 41 \mathrm{~B}^{\mathrm{ix}}$ & $2.751(3)$ & $\mathrm{C} 4 \mathrm{~B}-\mathrm{C} 5 \mathrm{~B}$ & $1.366(7)$ \\
\hline $\mathrm{K} 3-\mathrm{O} 42 \mathrm{~B}^{\mathrm{ix}}$ & $3.076(4)$ & $\mathrm{C} 5 \mathrm{~A}-\mathrm{C} 6 \mathrm{~A}$ & $1.394(6)$ \\
\hline $\mathrm{K} 3-\mathrm{O} 42 \mathrm{~A}^{\mathrm{vii}}$ & $2.938(3)$ & $\mathrm{C} 5 \mathrm{~B}-\mathrm{C} 6 \mathrm{~B}$ & $1.389(6)$ \\
\hline $\mathrm{K} 3-\mathrm{O} 43 \mathrm{~A}^{\mathrm{vii}}$ & $3.027(3)$ & $\mathrm{C} 2 \mathrm{~A}-\mathrm{H} 2 \mathrm{~A}$ & 0.9500 \\
\hline $\mathrm{S} 4 \mathrm{~A}-\mathrm{O} 41 \mathrm{~A}$ & $1.450(3)$ & $\mathrm{C} 2 \mathrm{~B}-\mathrm{H} 2 \mathrm{~B}$ & 0.9400 \\
\hline $\mathrm{S} 4 \mathrm{~A}-\mathrm{O} 42 \mathrm{~A}$ & $1.453(3)$ & $\mathrm{C} 3 \mathrm{~A}-\mathrm{H} 3 \mathrm{~A}$ & 0.9500 \\
\hline $\mathrm{S} 4 \mathrm{~A}-\mathrm{O} 43 \mathrm{~A}$ & $1.455(3)$ & $\mathrm{C} 3 \mathrm{~B}-\mathrm{H} 3 \mathrm{~B}$ & 0.9400 \\
\hline $\mathrm{S} 4 \mathrm{~A}-\mathrm{C} 4 \mathrm{~A}$ & $1.784(3)$ & $\mathrm{C} 5 \mathrm{~A}-\mathrm{H} 5 \mathrm{~A}$ & 0.9500 \\
\hline $\mathrm{S} 4 \mathrm{~B}-\mathrm{O} 41 \mathrm{~B}$ & $1.454(3)$ & $\mathrm{C} 5 \mathrm{~B}-\mathrm{H} 5 \mathrm{~B}$ & 0.9400 \\
\hline $\mathrm{S} 4 \mathrm{~B}-\mathrm{O} 42 \mathrm{~B}$ & $1.465(4)$ & $\mathrm{C} 6 \mathrm{~A}-\mathrm{H} 6 \mathrm{~A}$ & 0.9500 \\
\hline $\mathrm{S} 4 \mathrm{~B}-\mathrm{O} 43 \mathrm{~B}$ & $1.446(4)$ & $\mathrm{C} 6 \mathrm{~B}-\mathrm{H} 6 \mathrm{~B}$ & 0.9400 \\
\hline
\end{tabular}




\section{supplementary materials}

\begin{tabular}{|c|c|}
\hline $\mathrm{O} 41 \mathrm{~B}^{\mathrm{i}}-\mathrm{K} 1-\mathrm{O} 43 \mathrm{~B}$ & $110.25(9)$ \\
\hline $\mathrm{O} 41 \mathrm{~A}^{\mathrm{ii}}-\mathrm{K} 1-\mathrm{O} 43 \mathrm{~B}$ & $160.66(9)$ \\
\hline $\mathrm{O} 43 \mathrm{~A}^{\mathrm{ii}}-\mathrm{K} 1-\mathrm{O} 43 \mathrm{~B}$ & $152.59(10)$ \\
\hline 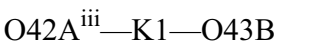 & $83.81(9)$ \\
\hline $\mathrm{O} 43 \mathrm{~B}-\mathrm{K} 1-\mathrm{O} 43 \mathrm{~B}^{\mathrm{iv}}$ & $71.84(10)$ \\
\hline $\mathrm{O} 41 \mathrm{~A}^{\mathrm{v}}-\mathrm{K} 1-\mathrm{O} 43 \mathrm{~B}$ & $95.63(10)$ \\
\hline 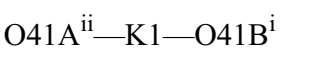 & $72.70(9)$ \\
\hline $\mathrm{O} 41 \mathrm{~B}^{\mathrm{i}}-\mathrm{K} 1-\mathrm{O} 43 \mathrm{~A}^{\mathrm{ii}}$ & $71.13(9)$ \\
\hline $\mathrm{O} 41 \mathrm{~B}^{\mathrm{i}}-\mathrm{K} 1-\mathrm{O} 42 \mathrm{~A}^{\mathrm{iii}}$ & $118.54(10)$ \\
\hline $\mathrm{O} 41 \mathrm{~B}^{\mathrm{i}}-\mathrm{K} 1-\mathrm{O} 43 \mathrm{~B}^{\mathrm{iv}}$ & $80.84(10)$ \\
\hline $\mathrm{O} 41 \mathrm{~A}^{\mathrm{v}}-\mathrm{K} 1-\mathrm{O} 41 \mathrm{~B}^{\mathrm{i}}$ & $139.11(11)$ \\
\hline $\mathrm{O} 41 \mathrm{~A}^{\mathrm{ii}}-\mathrm{K} 1-\mathrm{O} 43 \mathrm{~A}^{\mathrm{ii}}$ & $46.73(8)$ \\
\hline $\mathrm{O} 41 \mathrm{~A}^{\mathrm{ii}}-\mathrm{K} 1-\mathrm{O} 42 \mathrm{~A}^{\mathrm{iii}}$ & $112.09(9)$ \\
\hline $\mathrm{O} 41 \mathrm{~A}^{\mathrm{ii}}-\mathrm{K} 1-\mathrm{O} 43 \mathrm{~B}^{\mathrm{iv}}$ & $90.23(10)$ \\
\hline $\mathrm{O} 41 \mathrm{~A}^{\mathrm{ii}}-\mathrm{K} 1-\mathrm{O} 41 \mathrm{~A}^{\mathrm{v}}$ & $72.92(10)$ \\
\hline $\mathrm{O} 42 \mathrm{~A}^{\mathrm{iii}}-\mathrm{K} 1-\mathrm{O} 43 \mathrm{~A}^{\mathrm{ii}}$ & $72.58(9)$ \\
\hline $\mathrm{O} 43 \mathrm{~A}^{\mathrm{ii}}-\mathrm{K} 1-\mathrm{O} 43 \mathrm{~B}^{\mathrm{iv}}$ & $133.53(10)$ \\
\hline $\mathrm{O} 41 \mathrm{~A}^{\mathrm{v}}-\mathrm{K} 1-\mathrm{O} 43 \mathrm{~A}^{\mathrm{ii}}$ & $99.88(9)$ \\
\hline $\mathrm{O} 42 \mathrm{~A}^{\mathrm{iii}}-\mathrm{K} 1-\mathrm{O} 43 \mathrm{~B}^{\mathrm{iv}}$ & $153.45(10)$ \\
\hline $\mathrm{O} 41 \mathrm{~A}^{\mathrm{v}}-\mathrm{K} 1-\mathrm{O} 42 \mathrm{~A}^{\mathrm{iii}}$ & $94.61(10)$ \\
\hline $\mathrm{O} 41 \mathrm{~A}^{\mathrm{v}}-\mathrm{K} 1-\mathrm{O} 43 \mathrm{~B}^{\mathrm{iv}}$ & $77.83(9)$ \\
\hline $\mathrm{O} 1 \mathrm{~W}-\mathrm{K} 2-\mathrm{O} 2 \mathrm{~W}$ & $178.76(17)$ \\
\hline $\mathrm{O} 1 \mathrm{~W}-\mathrm{K} 2-\mathrm{O} 43 \mathrm{~A}^{\mathrm{ii}}$ & $101.02(12)$ \\
\hline $\mathrm{O} 1 \mathrm{~W}-\mathrm{K} 2-\mathrm{O} 42 \mathrm{~A}^{\mathrm{iii}}$ & $105.61(11)$ \\
\hline $\mathrm{O} 1 \mathrm{~W}-\mathrm{K} 2-\mathrm{O} 43 \mathrm{~A}^{\mathrm{vi}}$ & $101.02(12)$ \\
\hline $\mathrm{O} 1 \mathrm{~W}-\mathrm{K} 2-\mathrm{O} 42 \mathrm{~A}^{\mathrm{vii}}$ & $105.61(11)$ \\
\hline $\mathrm{O} 2 \mathrm{~W}-\mathrm{K} 2-\mathrm{O} 43 \mathrm{~A}^{\mathrm{ii}}$ & $79.84(9)$ \\
\hline $\mathrm{O} 2 \mathrm{~W}-\mathrm{K} 2-\mathrm{O} 42 \mathrm{~A}^{\mathrm{iii}}$ & $73.61(8)$ \\
\hline $\mathrm{O} 2 \mathrm{~W}-\mathrm{K} 2-\mathrm{O} 43 \mathrm{~A}^{\mathrm{vi}}$ & $79.84(9)$ \\
\hline $\mathrm{O} 2 \mathrm{~W}-\mathrm{K} 2-\mathrm{O} 42 \mathrm{~A}^{\mathrm{vii}}$ & $73.61(8)$ \\
\hline $\mathrm{O} 42 \mathrm{~A}^{\mathrm{iii}}-\mathrm{K} 2-\mathrm{O} 43 \mathrm{~A}^{\mathrm{ii}}$ & $81.09(9)$ \\
\hline $\mathrm{O} 43 \mathrm{~A}^{\mathrm{ii}}-\mathrm{K} 2-\mathrm{O} 43 \mathrm{~A}^{\mathrm{vi}}$ & $89.26(10)$ \\
\hline $\mathrm{O} 42 \mathrm{~A}^{\mathrm{vii}}-\mathrm{K} 2-\mathrm{O} 43 \mathrm{~A}^{\mathrm{ii}}$ & $152.93(10)$ \\
\hline $\mathrm{O} 42 \mathrm{~A}^{\mathrm{iii}}-\mathrm{K} 2-\mathrm{O} 43 \mathrm{~A}^{\mathrm{vi}}$ & $152.93(10)$ \\
\hline $\mathrm{O} 42 \mathrm{~A}^{\mathrm{iii}}-\mathrm{K} 2-\mathrm{O} 42 \mathrm{~A}^{\mathrm{vii}}$ & $96.15(10)$ \\
\hline $\mathrm{O} 42 \mathrm{~A}^{\mathrm{vii}}-\mathrm{K} 2-\mathrm{O} 43 \mathrm{~A}^{\mathrm{vi}}$ & $81.09(9)$ \\
\hline $\mathrm{S} 4 \mathrm{~B}-\mathrm{K} 3-\mathrm{O} 2 \mathrm{~W}$ & $78.47(5)$ \\
\hline $\mathrm{S} 4 \mathrm{~B}-\mathrm{K} 3-\mathrm{O} 41 \mathrm{~B}$ & $23.63(6)$ \\
\hline $\mathrm{S} 4 \mathrm{~B}-\mathrm{K} 3-\mathrm{O} 42 \mathrm{~B}$ & $24.96(7)$ \\
\hline $\mathrm{S} 4 \mathrm{~B}-\mathrm{K} 3-\mathrm{O} 42 \mathrm{~A}^{\mathrm{iii}}$ & $70.87(6)$ \\
\hline $\mathrm{S} 4 \mathrm{~B}-\mathrm{K} 3-\mathrm{O} 43 \mathrm{~A}^{\mathrm{iii}}$ & $85.56(6)$ \\
\hline
\end{tabular}

\begin{tabular}{|c|c|}
\hline $\mathrm{O} 41 \mathrm{~B}^{\mathrm{ix}}-\mathrm{K} 3-\mathrm{O} 42 \mathrm{~B}^{\mathrm{ix}}$ & $48.53(9)$ \\
\hline $\mathrm{O} 41 \mathrm{~B}^{\mathrm{ix}}-\mathrm{K} 3-\mathrm{O} 42 \mathrm{~A}^{\mathrm{vii}}$ & $75.26(9)$ \\
\hline $\mathrm{O} 41 \mathrm{~B}^{\mathrm{ix}}-\mathrm{K} 3-\mathrm{O} 43 \mathrm{~A}^{\mathrm{vii}}$ & $71.01(8)$ \\
\hline $\mathrm{O} 42 \mathrm{~A}^{\mathrm{vii}}-\mathrm{K} 3-\mathrm{O} 42 \mathrm{~B}^{\mathrm{ix}}$ & $72.09(8)$ \\
\hline $\mathrm{O} 42 \mathrm{~B}^{\mathrm{ix}}-\mathrm{K} 3-\mathrm{O} 43 \mathrm{~A}^{\mathrm{vii}}$ & $103.14(9)$ \\
\hline $\mathrm{O} 42 \mathrm{~A}^{\mathrm{vii}}-\mathrm{K} 3-\mathrm{O} 43 \mathrm{~A}^{\mathrm{vii}}$ & $47.64(8)$ \\
\hline $\mathrm{O} 41 \mathrm{~A}-\mathrm{S} 4 \mathrm{~A}-\mathrm{O} 42 \mathrm{~A}$ & $112.82(19)$ \\
\hline $\mathrm{O} 41 \mathrm{~A}-\mathrm{S} 4 \mathrm{~A}-\mathrm{O} 43 \mathrm{~A}$ & $112.72(19)$ \\
\hline $\mathrm{O} 41 \mathrm{~A}-\mathrm{S} 4 \mathrm{~A}-\mathrm{C} 4 \mathrm{~A}$ & $106.33(17)$ \\
\hline $\mathrm{O} 42 \mathrm{~A}-\mathrm{S} 4 \mathrm{~A}-\mathrm{O} 43 \mathrm{~A}$ & $111.95(18)$ \\
\hline $\mathrm{O} 42 \mathrm{~A}-\mathrm{S} 4 \mathrm{~A}-\mathrm{C} 4 \mathrm{~A}$ & $105.28(17)$ \\
\hline $\mathrm{O} 43 \mathrm{~A}-\mathrm{S} 4 \mathrm{~A}-\mathrm{C} 4 \mathrm{~A}$ & $107.12(18)$ \\
\hline $\mathrm{K} 3-\mathrm{S} 4 \mathrm{~B}-\mathrm{O} 41 \mathrm{~B}$ & $49.33(13)$ \\
\hline $\mathrm{K} 3-\mathrm{S} 4 \mathrm{~B}-\mathrm{O} 42 \mathrm{~B}$ & $62.39(13)$ \\
\hline $\mathrm{K} 3-\mathrm{S} 4 \mathrm{~B}-\mathrm{O} 43 \mathrm{~B}$ & $129.77(13)$ \\
\hline $\mathrm{K} 3-\mathrm{S} 4 \mathrm{~B}-\mathrm{C} 4 \mathrm{~B}$ & $122.20(14)$ \\
\hline $\mathrm{O} 41 \mathrm{~B}-\mathrm{S} 4 \mathrm{~B}-\mathrm{O} 42 \mathrm{~B}$ & $111.53(19)$ \\
\hline $\mathrm{O} 41 \mathrm{~B}-\mathrm{S} 4 \mathrm{~B}-\mathrm{O} 43 \mathrm{~B}$ & $114.83(19)$ \\
\hline $\mathrm{O} 41 \mathrm{~B}-\mathrm{S} 4 \mathrm{~B}-\mathrm{C} 4 \mathrm{~B}$ & $105.87(18)$ \\
\hline $\mathrm{O} 42 \mathrm{~B}-\mathrm{S} 4 \mathrm{~B}-\mathrm{O} 43 \mathrm{~B}$ & $111.0(2)$ \\
\hline $\mathrm{O} 42 \mathrm{~B}-\mathrm{S} 4 \mathrm{~B}-\mathrm{C} 4 \mathrm{~B}$ & $105.19(18)$ \\
\hline $\mathrm{O} 43 \mathrm{~B}-\mathrm{S} 4 \mathrm{~B}-\mathrm{C} 4 \mathrm{~B}$ & $107.79(18)$ \\
\hline $\mathrm{K} 2-\mathrm{O} 2 \mathrm{~W}-\mathrm{K} 3$ & $80.85(11)$ \\
\hline $\mathrm{K} 2-\mathrm{O} 2 \mathrm{~W}-\mathrm{K} 3^{\mathrm{i}}$ & $85.61(12)$ \\
\hline $\mathrm{K} 3-\mathrm{O} 2 \mathrm{~W}-\mathrm{K} 3^{\mathrm{i}}$ & $166.45(17)$ \\
\hline $\mathrm{K} 1^{\mathrm{x}}-\mathrm{O} 41 \mathrm{~A}-\mathrm{S} 4 \mathrm{~A}$ & $99.57(16)$ \\
\hline $\mathrm{K} 1^{\mathrm{xi}}-\mathrm{O} 41 \mathrm{~A}-\mathrm{S} 4 \mathrm{~A}$ & $158.5(2)$ \\
\hline $\mathrm{K} 1^{\mathrm{x}}-\mathrm{O} 41 \mathrm{~A}-\mathrm{K} 1^{\mathrm{xi}}$ & $85.21(9)$ \\
\hline $\mathrm{K} 3-\mathrm{O} 41 \mathrm{~B}-\mathrm{S} 4 \mathrm{~B}$ & $107.04(16)$ \\
\hline $\mathrm{K} 1^{\mathrm{viii}}-\mathrm{O} 41 \mathrm{~B}-\mathrm{K} 3$ & $111.44(10)$ \\
\hline $\mathrm{K} 1^{\mathrm{viii}}-\mathrm{O} 41 \mathrm{~B}-\mathrm{S} 4 \mathrm{~B}$ & $133.48(19)$ \\
\hline $\mathrm{K} 1^{\mathrm{xii}}-\mathrm{O} 42 \mathrm{~A}-\mathrm{S} 4 \mathrm{~A}$ & $118.71(18)$ \\
\hline $\mathrm{K} 2^{\mathrm{xii}}-\mathrm{O} 42 \mathrm{~A}-\mathrm{S} 4 \mathrm{~A}$ & $132.77(17)$ \\
\hline $\mathrm{K} 3{ }^{\mathrm{xii}}-\mathrm{O} 42 \mathrm{~A}-\mathrm{S} 4 \mathrm{~A}$ & $100.58(15)$ \\
\hline $\mathrm{K} 1^{\mathrm{xii}}-\mathrm{O} 42 \mathrm{~A}-\mathrm{K} 2^{\mathrm{xii}}$ & $100.58(10)$ \\
\hline $\mathrm{K} 1^{\mathrm{xii}}-\mathrm{O} 42 \mathrm{~A}-\mathrm{K} 3^{\mathrm{xii}}$ & $113.15(10)$ \\
\hline $\mathrm{K} 2^{\mathrm{xii}}-\mathrm{O} 42 \mathrm{~A}-\mathrm{K} 3^{\mathrm{xii}}$ & $85.52(10)$ \\
\hline $\mathrm{K} 3-\mathrm{O} 42 \mathrm{~B}-\mathrm{S} 4 \mathrm{~B}$ & $92.65(15)$ \\
\hline $\mathrm{K} 3^{\mathrm{xii}}-\mathrm{O} 43 \mathrm{~A}-\mathrm{S} 4 \mathrm{~A}$ & $96.74(14)$ \\
\hline $\mathrm{K} 1^{\mathrm{x}}-\mathrm{O} 43 \mathrm{~A}-\mathrm{S} 4 \mathrm{~A}$ & $98.62(15)$ \\
\hline $\mathrm{K} 2^{\mathrm{x}}-\mathrm{O} 43 \mathrm{~A}-\mathrm{S} 4 \mathrm{~A}$ & $163.26(19)$ \\
\hline
\end{tabular}




\section{supplementary materials}

\begin{tabular}{|c|c|}
\hline $\mathrm{S} 4 \mathrm{~B}-\mathrm{K} 3-\mathrm{O} 2 \mathrm{~W}^{\mathrm{viii}}$ & $94.10(6)$ \\
\hline $\mathrm{S} 4 \mathrm{~B}-\mathrm{K} 3-\mathrm{S} 4 \mathrm{~B}^{\mathrm{ix}}$ & $111.64(4)$ \\
\hline $\mathrm{S} 4 \mathrm{~B}-\mathrm{K} 3-\mathrm{O} 41 \mathrm{~B}^{\mathrm{ix}}$ & $121.69(7)$ \\
\hline $\mathrm{S} 4 \mathrm{~B}-\mathrm{K} 3-\mathrm{O} 42 \mathrm{~B}^{\mathrm{ix}}$ & $96.07(7)$ \\
\hline $\mathrm{S} 4 \mathrm{~B}-\mathrm{K} 3-\mathrm{O} 42 \mathrm{~A}^{\mathrm{vii}}$ & $145.18(7)$ \\
\hline $\mathrm{S} 4 \mathrm{~B}-\mathrm{K} 3-\mathrm{O} 43 \mathrm{~A}^{\mathrm{vii}}$ & $160.55(7)$ \\
\hline $\mathrm{O} 2 \mathrm{~W}-\mathrm{K} 3-\mathrm{O} 41 \mathrm{~B}$ & $101.53(8)$ \\
\hline $\mathrm{O} 2 \mathrm{~W}-\mathrm{K} 3-\mathrm{O} 42 \mathrm{~B}$ & $54.96(9)$ \\
\hline $\mathrm{O} 2 \mathrm{~W}-\mathrm{K} 3-\mathrm{O} 42 \mathrm{~A}^{\mathrm{iii}}$ & $67.86(8)$ \\
\hline $\mathrm{O} 2 \mathrm{~W}-\mathrm{K} 3-\mathrm{O} 43 \mathrm{~A}^{\mathrm{iii}}$ & $115.20(9)$ \\
\hline $\mathrm{O} 2 \mathrm{~W}-\mathrm{K} 3-\mathrm{O} 2 \mathrm{~W}^{\mathrm{viii}}$ & $166.45(13)$ \\
\hline $\mathrm{S} 4 \mathrm{~B}^{\mathrm{ix}}-\mathrm{K} 3-\mathrm{O} 2 \mathrm{~W}$ & $78.47(5)$ \\
\hline $\mathrm{O} 2 \mathrm{~W}-\mathrm{K} 3-\mathrm{O} 41 \mathrm{~B}^{\mathrm{ix}}$ & $101.53(8)$ \\
\hline $\mathrm{O} 2 \mathrm{~W}-\mathrm{K} 3-\mathrm{O} 42 \mathrm{~B}^{\mathrm{ix}}$ & $54.96(9)$ \\
\hline $\mathrm{O} 2 \mathrm{~W}-\mathrm{K} 3-\mathrm{O} 42 \mathrm{~A}^{\mathrm{vii}}$ & $67.86(8)$ \\
\hline $\mathrm{O} 2 \mathrm{~W}-\mathrm{K} 3-\mathrm{O} 43 \mathrm{~A}^{\mathrm{vii}}$ & $115.20(9)$ \\
\hline $\mathrm{O} 41 \mathrm{~B}-\mathrm{K} 3-\mathrm{O} 42 \mathrm{~B}$ & $48.53(9)$ \\
\hline $\mathrm{O} 41 \mathrm{~B}-\mathrm{K} 3-\mathrm{O} 42 \mathrm{~A}^{\mathrm{iii}}$ & $75.26(9)$ \\
\hline $\mathrm{O} 41 \mathrm{~B}-\mathrm{K} 3-\mathrm{O} 43 \mathrm{~A}^{\mathrm{iii}}$ & $71.01(8)$ \\
\hline $\mathrm{O} 2 \mathrm{~W}^{\mathrm{viii}}-\mathrm{K} 3-\mathrm{O} 41 \mathrm{~B}$ & $72.58(8)$ \\
\hline $\mathrm{S} 4 \mathrm{~B}^{\mathrm{ix}}-\mathrm{K} 3-\mathrm{O} 41 \mathrm{~B}$ & $121.69(7)$ \\
\hline $\mathrm{O} 41 \mathrm{~B}-\mathrm{K} 3-\mathrm{O} 41 \mathrm{~B}^{\mathrm{ix}}$ & $121.19(10)$ \\
\hline $\mathrm{O} 41 \mathrm{~B}-\mathrm{K} 3-\mathrm{O} 42 \mathrm{~B}^{\mathrm{ix}}$ & $113.82(9)$ \\
\hline $\mathrm{O} 41 \mathrm{~B}-\mathrm{K} 3-\mathrm{O} 42 \mathrm{~A}^{\mathrm{vii}}$ & $162.77(10)$ \\
\hline $\mathrm{O} 41 \mathrm{~B}-\mathrm{K} 3-\mathrm{O} 43 \mathrm{~A}^{\mathrm{vii}}$ & $138.51(9)$ \\
\hline 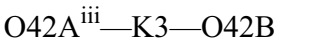 & $72.09(8)$ \\
\hline $\mathrm{O} 42 \mathrm{~B}-\mathrm{K} 3-\mathrm{O} 43 \mathrm{~A}^{\mathrm{iii}}$ & $103.14(9)$ \\
\hline $\mathrm{O} 2 \mathrm{~W}^{\mathrm{viii}}-\mathrm{K} 3-\mathrm{O} 42 \mathrm{~B}$ & $115.52(10)$ \\
\hline $\mathrm{S} 4 \mathrm{~B}^{\mathrm{ix}}-\mathrm{K} 3-\mathrm{O} 42 \mathrm{~B}$ & $96.07(7)$ \\
\hline $\mathrm{O} 41 \mathrm{~B}^{\mathrm{ix}}-\mathrm{K} 3-\mathrm{O} 42 \mathrm{~B}$ & $113.82(9)$ \\
\hline $\mathrm{O} 42 \mathrm{~B}-\mathrm{K} 3-\mathrm{O} 42 \mathrm{~B}^{\mathrm{ix}}$ & $75.45(9)$ \\
\hline 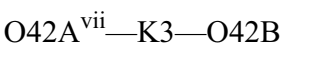 & $122.82(10)$ \\
\hline $\mathrm{O} 42 \mathrm{~B}-\mathrm{K} 3-\mathrm{O} 43 \mathrm{~A}^{\mathrm{vii}}$ & $169.11(9)$ \\
\hline $\mathrm{O} 42 \mathrm{~A}^{\mathrm{iii}}-\mathrm{K} 3-\mathrm{O} 43 \mathrm{~A}^{\mathrm{iii}}$ & $47.64(8)$ \\
\hline $\mathrm{O} 2 \mathrm{~W}^{\mathrm{viii}}-\mathrm{K} 3-\mathrm{O} 42 \mathrm{~A}^{\mathrm{iii}}$ & $120.66(8)$ \\
\hline $\mathrm{S} 4 \mathrm{~B}^{\mathrm{ix}}-\mathrm{K} 3-\mathrm{O} 42 \mathrm{~A}^{\mathrm{iii}}$ & $145.18(7)$ \\
\hline $\mathrm{O} 41 \mathrm{~B}^{\mathrm{ix}}-\mathrm{K} 3-\mathrm{O} 42 \mathrm{~A}^{\mathrm{iii}}$ & $162.77(10)$ \\
\hline $\mathrm{O} 42 \mathrm{~A}^{\mathrm{iii}}-\mathrm{K} 3-\mathrm{O} 42 \mathrm{~B}^{\mathrm{ix}}$ & $122.82(10)$ \\
\hline $\mathrm{O} 42 \mathrm{~A}^{\mathrm{iii}}-\mathrm{K} 3-\mathrm{O} 42 \mathrm{~A}^{\mathrm{vii}}$ & $87.93(9)$ \\
\hline $\mathrm{O} 42 \mathrm{~A}^{\mathrm{iii}}-\mathrm{K} 3-\mathrm{O} 43 \mathrm{~A}^{\mathrm{vii}}$ & $100.51(9)$ \\
\hline $\mathrm{O} 2 \mathrm{~W}^{\mathrm{viii}}-\mathrm{K} 3-\mathrm{O} 43 \mathrm{~A}^{\mathrm{iii}}$ & $75.00(9)$ \\
\hline
\end{tabular}

\begin{tabular}{|c|c|}
\hline $\mathrm{K} 1^{\mathrm{x}}-\mathrm{O} 43 \mathrm{~A}-\mathrm{K} 3^{\mathrm{xii}}$ & $95.55(9)$ \\
\hline $\mathrm{K} 2^{\mathrm{x}}-\mathrm{O} 43 \mathrm{~A}-\mathrm{K} 3^{\mathrm{xii}}$ & $84.90(9)$ \\
\hline $\mathrm{K} 1^{\mathrm{x}}-\mathrm{O} 43 \mathrm{~A}-\mathrm{K} 2^{\mathrm{x}}$ & $97.79(9)$ \\
\hline $\mathrm{K} 1-\mathrm{O} 43 \mathrm{~B}-\mathrm{S} 4 \mathrm{~B}$ & $104.92(17)$ \\
\hline $\mathrm{K} 1-\mathrm{O} 43 \mathrm{~B}-\mathrm{K} 1^{\mathrm{xiii}}$ & $87.96(9)$ \\
\hline $\mathrm{K} 1^{\mathrm{xiii}}-\mathrm{O} 43 \mathrm{~B}-\mathrm{S} 4 \mathrm{~B}$ & $139.4(2)$ \\
\hline $\mathrm{H} 11 \mathrm{~W}-\mathrm{O} 1 \mathrm{~W}-\mathrm{H} 12 \mathrm{~W}$ & 113.00 \\
\hline $\mathrm{K} 2-\mathrm{O} 1 \mathrm{~W}-\mathrm{H} 11 \mathrm{~W}$ & 117.00 \\
\hline $\mathrm{K} 2-\mathrm{O} 1 \mathrm{~W}-\mathrm{H} 12 \mathrm{~W}$ & 130.00 \\
\hline $\mathrm{K} 2-\mathrm{O} 2 \mathrm{~W}-\mathrm{H} 2 \mathrm{~W}$ & 111.00 \\
\hline $\mathrm{K} 3-\mathrm{O} 2 \mathrm{~W}-\mathrm{H} 2 \mathrm{~W}^{\mathrm{ix}}$ & 65.00 \\
\hline $\mathrm{K} 2-\mathrm{O} 2 \mathrm{~W}-\mathrm{H} 2 \mathrm{~W}^{\mathrm{ix}}$ & 111.00 \\
\hline $\mathrm{K} 3-\mathrm{O} 2 \mathrm{~W}-\mathrm{H} 2 \mathrm{~W}$ & 65.00 \\
\hline $\mathrm{K} 3{ }^{\mathrm{i}}-\mathrm{O} 2 \mathrm{~W}-\mathrm{H} 2 \mathrm{~W}^{\mathrm{ix}}$ & 121.00 \\
\hline $\mathrm{K} 3{ }^{\mathrm{i}}-\mathrm{O} 2 \mathrm{~W}-\mathrm{H} 2 \mathrm{~W}$ & 121.00 \\
\hline $\mathrm{H} 2 \mathrm{~W}-\mathrm{O} 2 \mathrm{~W}-\mathrm{H} 2 \mathrm{~W}^{\mathrm{ix}}$ & 106.00 \\
\hline $\mathrm{H} 3 \mathrm{~W}-\mathrm{O} 3 \mathrm{~W}-\mathrm{H} 3 \mathrm{~W}^{\mathrm{ix}}$ & 104.00 \\
\hline $\mathrm{H} 41 \mathrm{~W}-\mathrm{O} 4 \mathrm{~W}-\mathrm{H} 42 \mathrm{~W}$ & 106.00 \\
\hline $\mathrm{C} 2 \mathrm{~A}-\mathrm{C} 1 \mathrm{~A}-\mathrm{C} 6 \mathrm{~A}$ & $117.2(4)$ \\
\hline $\mathrm{C} 1 \mathrm{~B}-\mathrm{C} 1 \mathrm{~A}-\mathrm{C} 6 \mathrm{~A}$ & $121.2(4)$ \\
\hline $\mathrm{C} 1 \mathrm{~B}-\mathrm{C} 1 \mathrm{~A}-\mathrm{C} 2 \mathrm{~A}$ & $121.6(4)$ \\
\hline $\mathrm{C} 1 \mathrm{~A}-\mathrm{C} 1 \mathrm{~B}-\mathrm{C} 6 \mathrm{~B}$ & $120.9(4)$ \\
\hline $\mathrm{C} 1 \mathrm{~A}-\mathrm{C} 1 \mathrm{~B}-\mathrm{C} 2 \mathrm{~B}$ & $122.1(4)$ \\
\hline $\mathrm{C} 2 \mathrm{~B}-\mathrm{C} 1 \mathrm{~B}-\mathrm{C} 6 \mathrm{~B}$ & $117.1(4)$ \\
\hline $\mathrm{C} 1 \mathrm{~A}-\mathrm{C} 2 \mathrm{~A}-\mathrm{C} 3 \mathrm{~A}$ & $121.4(4)$ \\
\hline $\mathrm{C} 1 \mathrm{~B}-\mathrm{C} 2 \mathrm{~B}-\mathrm{C} 3 \mathrm{~B}$ & $122.1(4)$ \\
\hline $\mathrm{C} 2 \mathrm{~A}-\mathrm{C} 3 \mathrm{~A}-\mathrm{C} 4 \mathrm{~A}$ & $119.8(4)$ \\
\hline $\mathrm{C} 2 \mathrm{~B}-\mathrm{C} 3 \mathrm{~B}-\mathrm{C} 4 \mathrm{~B}$ & $119.1(4)$ \\
\hline $\mathrm{S} 4 \mathrm{~A}-\mathrm{C} 4 \mathrm{~A}-\mathrm{C} 3 \mathrm{~A}$ & $119.6(3)$ \\
\hline $\mathrm{S} 4 \mathrm{~A}-\mathrm{C} 4 \mathrm{~A}-\mathrm{C} 5 \mathrm{~A}$ & $120.5(3)$ \\
\hline $\mathrm{C} 3 \mathrm{~A}-\mathrm{C} 4 \mathrm{~A}-\mathrm{C} 5 \mathrm{~A}$ & $119.9(3)$ \\
\hline $\mathrm{S} 4 \mathrm{~B}-\mathrm{C} 4 \mathrm{~B}-\mathrm{C} 3 \mathrm{~B}$ & $120.5(3)$ \\
\hline $\mathrm{S} 4 \mathrm{~B}-\mathrm{C} 4 \mathrm{~B}-\mathrm{C} 5 \mathrm{~B}$ & $119.5(3)$ \\
\hline $\mathrm{C} 3 \mathrm{~B}-\mathrm{C} 4 \mathrm{~B}-\mathrm{C} 5 \mathrm{~B}$ & $120.0(4)$ \\
\hline $\mathrm{C} 4 \mathrm{~A}-\mathrm{C} 5 \mathrm{~A}-\mathrm{C} 6 \mathrm{~A}$ & $119.9(4)$ \\
\hline $\mathrm{C} 4 \mathrm{~B}-\mathrm{C} 5 \mathrm{~B}-\mathrm{C} 6 \mathrm{~B}$ & $120.0(5)$ \\
\hline $\mathrm{C} 1 \mathrm{~A}-\mathrm{C} 6 \mathrm{~A}-\mathrm{C} 5 \mathrm{~A}$ & $121.7(5)$ \\
\hline $\mathrm{C} 1 \mathrm{~B}-\mathrm{C} 6 \mathrm{~B}-\mathrm{C} 5 \mathrm{~B}$ & $121.7(5)$ \\
\hline $\mathrm{C} 1 \mathrm{~A}-\mathrm{C} 2 \mathrm{~A}-\mathrm{H} 2 \mathrm{~A}$ & 118.00 \\
\hline $\mathrm{C} 3 \mathrm{~A}-\mathrm{C} 2 \mathrm{~A}-\mathrm{H} 2 \mathrm{~A}$ & 121.00 \\
\hline $\mathrm{C} 1 \mathrm{~B}-\mathrm{C} 2 \mathrm{~B}-\mathrm{H} 2 \mathrm{~B}$ & 118.00 \\
\hline
\end{tabular}




\section{supplementary materials}

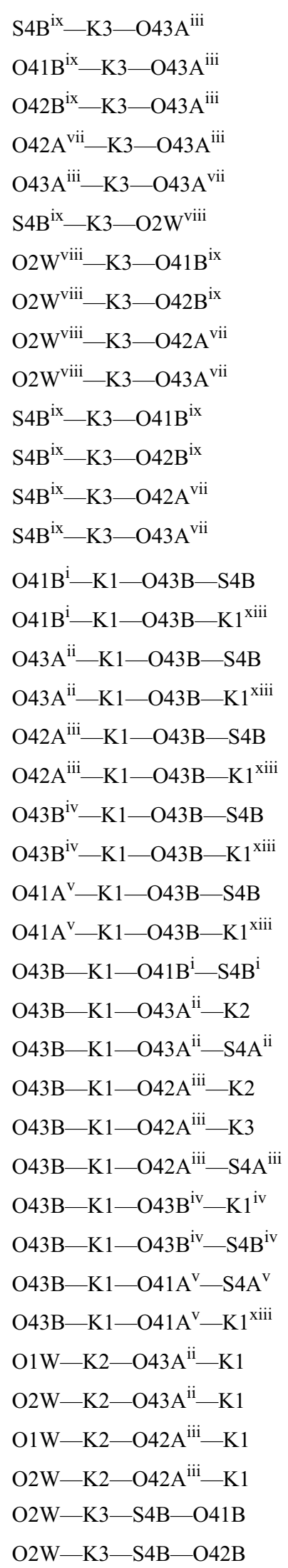

$160.55(7)$

$138.51(9)$

$169.11(9)$

$100.51(9)$

$76.15(9)$

$94.10(6)$

$72.58(8)$

$115.52(10)$

$120.66(8)$

$75.00(9)$

$23.63(6)$

$24.96(7)$

$70.87(6)$

$85.56(6)$

$-63.28(18)$

155.80 (9)

$24.6(3)$

$-116.35(19)$

54.89 (16)

$-86.03(10)$

-135.85 (18)

$83.23(10)$

148.94 (16)

$8.02(10)$

$-92.7(2)$

$10.8(2)$

$-172.48(16)$

-145.49 (11)

$-55.87(11)$

61.65 (17)

$-165.58(11)$

$-54.9(3)$

$96.8(5)$

$-7.24(9)$

$125.43(12)$

$-53.67(9)$

$-121.86(13)$

59.14 (10)

$167.29(17)$

$-18.15(16)$

$\begin{array}{ll}\mathrm{C} 3 \mathrm{~B}-\mathrm{C} 2 \mathrm{~B}-\mathrm{H} 2 \mathrm{~B} & 120.00 \\ \mathrm{C} 4 \mathrm{~A}-\mathrm{C} 3 \mathrm{~A}-\mathrm{H} 3 \mathrm{~A} & 119.00 \\ \mathrm{C} 2 \mathrm{~A}-\mathrm{C} 3 \mathrm{~A}-\mathrm{H} 3 \mathrm{~A} & 121.00 \\ \mathrm{C} 2 \mathrm{~B}-\mathrm{C} 3 \mathrm{~B}-\mathrm{H} 3 \mathrm{~B} & 121.00 \\ \mathrm{C} 4 \mathrm{~B}-\mathrm{C} 3 \mathrm{~B}-\mathrm{H} 3 \mathrm{~B} & 120.00 \\ \mathrm{C} 4 \mathrm{~A}-\mathrm{C} 5 \mathrm{~A}-\mathrm{H} 5 \mathrm{~A} & 119.00 \\ \mathrm{C} 6 \mathrm{~A}-\mathrm{C} 5 \mathrm{~A}-\mathrm{H} 5 \mathrm{~A} & 121.00 \\ \mathrm{C} 6 \mathrm{~B}-\mathrm{C} 5 \mathrm{~B}-\mathrm{H} 5 \mathrm{~B} & 121.00 \\ \mathrm{C} 4 \mathrm{~B}-\mathrm{C} 5 \mathrm{~B}-\mathrm{H} 5 \mathrm{~B} & 119.00 \\ \mathrm{C} 1 \mathrm{~A}-\mathrm{C} 6 \mathrm{~A}-\mathrm{H} 6 \mathrm{~A} & 118.00 \\ \mathrm{C} 5 \mathrm{~A}-\mathrm{C} 6 \mathrm{~A}-\mathrm{H} 6 \mathrm{~A} & 120.00 \\ \mathrm{C} 5 \mathrm{~B}-\mathrm{C} 6 \mathrm{~B}-\mathrm{H} 6 \mathrm{~B} & 120.00 \\ \mathrm{C} 1 \mathrm{~B}-\mathrm{C} 6 \mathrm{~B}-\mathrm{H} 6 \mathrm{~B} & 118.00\end{array}$

$\mathrm{S} 4 \mathrm{~B}-\mathrm{K} 3-\mathrm{O} 42 \mathrm{~A}^{\mathrm{iii}-\mathrm{K} 1 \quad 36.28(8)}$

$\mathrm{S} 4 \mathrm{~B}-\mathrm{K} 3-\mathrm{O} 42 \mathrm{~A}^{\mathrm{iii}}-\mathrm{K} 2 \quad 135.88(8)$

$\mathrm{O} 2 \mathrm{~W}-\mathrm{K} 3-\mathrm{O} 42 \mathrm{~A}^{\mathrm{iii}-\mathrm{K} 1} \quad-48.71$ (11)

O41B-K3-O42A $\mathrm{A}^{\mathrm{iii}}-\mathrm{K} 1 \quad 60.57$ (11)

$\mathrm{O} 41 \mathrm{~B}-\mathrm{K} 3-\mathrm{O} 42 \mathrm{~A}^{\mathrm{iii}}-\mathrm{K} 2 \quad 160.18(10)$

O42B-K3-O42A ${ }^{\mathrm{iii}-\mathrm{K} 1} \quad 9.96(11)$

O42B-K3-O42A ${ }^{\mathrm{iii}-\mathrm{K} 2} \quad 109.57(10)$

$\mathrm{S} 4 \mathrm{~B}-\mathrm{K} 3-\mathrm{O} 42 \mathrm{~A}^{\mathrm{vii}}-\mathrm{K} 2 \quad-35.22(15)$

$\mathrm{O} 42 \mathrm{~B}-\mathrm{K} 3-\mathrm{O} 42 \mathrm{~A}^{\mathrm{vii}}-\mathrm{K} 2 \quad-51.07$ (12)

$\mathrm{O} 42 \mathrm{~A}-\mathrm{S} 4 \mathrm{~A}-\mathrm{O} 41 \mathrm{~A}-\mathrm{K} 1^{\mathrm{x}} \quad-112.87(16)$

$\mathrm{O} 42 \mathrm{~A}-\mathrm{S} 4 \mathrm{~A}-\mathrm{O} 41 \mathrm{~A}-\mathrm{K}^{\mathrm{xi}} \quad-11.5(6)$

O43A-S4A-O41A-K1 ${ }^{\mathrm{X}} \quad 15.15(19)$

$\mathrm{O} 43 \mathrm{~A}-\mathrm{S} 4 \mathrm{~A}-\mathrm{O} 41 \mathrm{~A}-\mathrm{K}^{\mathrm{xi}} \quad 116.5(5)$

$\mathrm{C} 4 \mathrm{~A}-\mathrm{S} 4 \mathrm{~A}-\mathrm{O} 41 \mathrm{~A}-\mathrm{K} 1^{\mathrm{X}} \quad 132.23(15)$

$\mathrm{C} 4 \mathrm{~A}-\mathrm{S} 4 \mathrm{~A}-\mathrm{O} 41 \mathrm{~A}-\mathrm{K} 1^{\mathrm{xi}} \quad-126.4(5)$

$\mathrm{O} 41 \mathrm{~A}-\mathrm{S} 4 \mathrm{~A}-\mathrm{O} 42 \mathrm{~A}-\mathrm{K} 1^{\mathrm{xii}} \quad-13.0(2)$

$\mathrm{O} 41 \mathrm{~A}-\mathrm{S} 4 \mathrm{~A}-\mathrm{O} 42 \mathrm{~A}-\mathrm{K} 2^{\mathrm{xii}} \quad-155.4$ (2)

$\mathrm{O} 41 \mathrm{~A}-\mathrm{S} 4 \mathrm{~A}-\mathrm{O} 42 \mathrm{~A}-\mathrm{K} 3^{\mathrm{xii}} \quad 110.94$ (17)

$\mathrm{O} 43 \mathrm{~A}-\mathrm{S} 4 \mathrm{~A}-\mathrm{O} 42 \mathrm{~A}-\mathrm{K} 1^{\mathrm{xii}} \quad-141.42(17)$

O43A-S4A-O42A-K2 $2^{\mathrm{xii}} \quad 76.2(3)$

O43A-S4A-O42A-K3 ${ }^{\mathrm{xi}} \quad-17.47$ (19)

$\mathrm{C} 4 \mathrm{~A}-\mathrm{S} 4 \mathrm{~A}-\mathrm{O} 42 \mathrm{~A}-\mathrm{K} 1^{\mathrm{xii}} \quad 102.53(18)$

$\mathrm{C} 4 \mathrm{~A}-\mathrm{S} 4 \mathrm{~A}-\mathrm{O} 42 \mathrm{~A}-\mathrm{K}^{\mathrm{xii}} \quad-39.8(3)$

$\mathrm{C} 4 \mathrm{~A}-\mathrm{S} 4 \mathrm{~A}-\mathrm{O} 42 \mathrm{~A}-\mathrm{K} 3^{\mathrm{xii}} \quad-133.52(14)$

O41A-S4A-O43A-K3 ${ }^{\mathrm{xii}} \quad-111.70(15)$

$\mathrm{O} 41 \mathrm{~A}-\mathrm{S} 4 \mathrm{~A}-\mathrm{O} 43 \mathrm{~A}-\mathrm{K}^{\mathrm{X}} \quad-15.01(18)$ 


\section{supplementary materials}

\begin{tabular}{|c|c|}
\hline $\mathrm{O} 2 \mathrm{~W}-\mathrm{K} 3-\mathrm{S} 4 \mathrm{~B}-\mathrm{O} 43 \mathrm{~B}$ & $77.0(2)$ \\
\hline $\mathrm{O} 2 \mathrm{~W}-\mathrm{K} 3-\mathrm{S} 4 \mathrm{~B}-\mathrm{C} 4 \mathrm{~B}$ & $-109.31(18)$ \\
\hline $\mathrm{O} 41 \mathrm{~B}-\mathrm{K} 3-\mathrm{S} 4 \mathrm{~B}-\mathrm{O} 42 \mathrm{~B}$ & $174.6(2)$ \\
\hline $\mathrm{O} 41 \mathrm{~B}-\mathrm{K} 3-\mathrm{S} 4 \mathrm{~B}-\mathrm{O} 43 \mathrm{~B}$ & $-90.3(2)$ \\
\hline $\mathrm{O} 41 \mathrm{~B}-\mathrm{K} 3-\mathrm{S} 4 \mathrm{~B}-\mathrm{C} 4 \mathrm{~B}$ & $83.4(2)$ \\
\hline $\mathrm{O} 42 \mathrm{~B}-\mathrm{K} 3-\mathrm{S} 4 \mathrm{~B}-\mathrm{O} 41 \mathrm{~B}$ & $-174.6(2)$ \\
\hline $\mathrm{O} 42 \mathrm{~B}-\mathrm{K} 3-\mathrm{S} 4 \mathrm{~B}-\mathrm{O} 43 \mathrm{~B}$ & $95.2(2)$ \\
\hline $\mathrm{O} 42 \mathrm{~B}-\mathrm{K} 3-\mathrm{S} 4 \mathrm{~B}-\mathrm{C} 4 \mathrm{~B}$ & $-91.2(2)$ \\
\hline $\mathrm{O} 42 \mathrm{~A}^{\mathrm{iii}}-\mathrm{K} 3-\mathrm{S} 4 \mathrm{~B}-\mathrm{O} 41 \mathrm{~B}$ & $96.94(16)$ \\
\hline $\mathrm{O} 42 \mathrm{~A}^{\mathrm{iii}}-\mathrm{K} 3-\mathrm{S} 4 \mathrm{~B}-\mathrm{O} 42 \mathrm{~B}$ & $-88.50(16)$ \\
\hline $\mathrm{O} 42 \mathrm{~A}^{\mathrm{iii}}-\mathrm{K} 3-\mathrm{S} 4 \mathrm{~B}-\mathrm{O} 43 \mathrm{~B}$ & $6.6(2)$ \\
\hline $\mathrm{O} 42 \mathrm{~A}^{\mathrm{iii}}-\mathrm{K} 3-\mathrm{S} 4 \mathrm{~B}-\mathrm{C} 4 \mathrm{~B}$ & $-179.66(18)$ \\
\hline 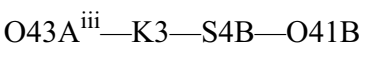 & $50.45(16)$ \\
\hline $\mathrm{O} 43 \mathrm{~A}^{\mathrm{iii}}-\mathrm{K} 3-\mathrm{S} 4 \mathrm{~B}-\mathrm{O} 42 \mathrm{~B}$ & $-135.00(15)$ \\
\hline $\mathrm{O} 43 \mathrm{~A}^{\mathrm{iii}}-\mathrm{K} 3-\mathrm{S} 4 \mathrm{~B}-\mathrm{O} 43 \mathrm{~B}$ & $-39.9(2)$ \\
\hline $\mathrm{O} 43 \mathrm{~A}^{\mathrm{iii}}-\mathrm{K} 3-\mathrm{S} 4 \mathrm{~B}-\mathrm{C} 4 \mathrm{~B}$ & $133.85(17)$ \\
\hline $\mathrm{O} 2 \mathrm{~W}^{\mathrm{vii}}-\mathrm{K} 3-\mathrm{S} 4 \mathrm{~B}-\mathrm{O} 41 \mathrm{~B}$ & $-24.14(17)$ \\
\hline $\mathrm{O} 2 \mathrm{~W}^{\mathrm{vii}}-\mathrm{K} 3-\mathrm{S} 4 \mathrm{~B}-\mathrm{O} 42 \mathrm{~B}$ & $150.41(16)$ \\
\hline $\mathrm{O} 2 \mathrm{~W}^{\mathrm{viii}}-\mathrm{K} 3-\mathrm{S} 4 \mathrm{~B}-\mathrm{O} 43 \mathrm{~B}$ & $-114.4(2)$ \\
\hline $\mathrm{O} 2 \mathrm{~W}^{\mathrm{viii}}-\mathrm{K} 3-\mathrm{S} 4 \mathrm{~B}-\mathrm{C} 4 \mathrm{~B}$ & $59.26(18)$ \\
\hline$S 4 B^{i x}-K 3-S 4 B-O 41 B$ & $-120.20(16)$ \\
\hline$S 4 B^{i x}-K 3-S 4 B-O 42 B$ & $54.36(14)$ \\
\hline $\mathrm{S} 4 \mathrm{~B}^{\mathrm{ix}}-\mathrm{K} 3-\mathrm{S} 4 \mathrm{~B}-\mathrm{O} 43 \mathrm{~B}$ & $149.50(19)$ \\
\hline$S 4 B^{i x}-K 3-S 4 B-C 4 B$ & $-36.80(17)$ \\
\hline $\mathrm{O} 41 \mathrm{~B}^{\mathrm{ix}}-\mathrm{K} 3-\mathrm{S} 4 \mathrm{~B}-\mathrm{O} 41 \mathrm{~B}$ & $-96.17(17)$ \\
\hline $\mathrm{O} 41 \mathrm{~B}^{\mathrm{ix}}-\mathrm{K} 3-\mathrm{S} 4 \mathrm{~B}-\mathrm{O} 42 \mathrm{~B}$ & $78.38(16)$ \\
\hline $\mathrm{O} 41 \mathrm{~B}^{\mathrm{ix}}-\mathrm{K} 3-\mathrm{S} 4 \mathrm{~B}-\mathrm{O} 43 \mathrm{~B}$ & $173.5(2)$ \\
\hline $\mathrm{O} 41 \mathrm{~B}^{\mathrm{ix}}-\mathrm{K} 3-\mathrm{S} 4 \mathrm{~B}-\mathrm{C} 4 \mathrm{~B}$ & $-12.77(19)$ \\
\hline $\mathrm{O} 42 \mathrm{~B}^{\mathrm{ix}}-\mathrm{K} 3-\mathrm{S} 4 \mathrm{~B}-\mathrm{O} 41 \mathrm{~B}$ & $-140.37(17)$ \\
\hline $\mathrm{O} 42 \mathrm{~B}^{\mathrm{ix}}-\mathrm{K} 3-\mathrm{S} 4 \mathrm{~B}-\mathrm{O} 42 \mathrm{~B}$ & $34.19(16)$ \\
\hline $\mathrm{O} 42 \mathrm{~B}^{\mathrm{ix}}-\mathrm{K} 3-\mathrm{S} 4 \mathrm{~B}-\mathrm{O} 43 \mathrm{~B}$ & $129.3(2)$ \\
\hline $\mathrm{O} 42 \mathrm{~B}^{\mathrm{ix}}-\mathrm{K} 3-\mathrm{S} 4 \mathrm{~B}-\mathrm{C} 4 \mathrm{~B}$ & $-56.97(18)$ \\
\hline $\mathrm{O} 42 \mathrm{~A}^{\mathrm{vii}}-\mathrm{K} 3-\mathrm{S} 4 \mathrm{~B}-\mathrm{O} 41 \mathrm{~B}$ & $152.50(19)$ \\
\hline $\mathrm{O} 42 \mathrm{~A}^{\mathrm{vii}}-\mathrm{K} 3-\mathrm{S} 4 \mathrm{~B}-\mathrm{O} 42 \mathrm{~B}$ & $-32.95(18)$ \\
\hline $\mathrm{O} 42 \mathrm{~A}^{\mathrm{vii}}-\mathrm{K} 3-\mathrm{S} 4 \mathrm{~B}-\mathrm{O} 43 \mathrm{~B}$ & $62.2(2)$ \\
\hline $\mathrm{O} 42 \mathrm{~A}^{\mathrm{vii}}-\mathrm{K} 3-\mathrm{S} 4 \mathrm{~B}-\mathrm{C} 4 \mathrm{~B}$ & $-124.1(2)$ \\
\hline $\mathrm{S} 4 \mathrm{~B}-\mathrm{K} 3-\mathrm{O} 2 \mathrm{~W}-\mathrm{K} 2$ & $-122.40(3)$ \\
\hline $\mathrm{O} 41 \mathrm{~B}-\mathrm{K} 3-\mathrm{O} 2 \mathrm{~W}-\mathrm{K} 2$ & $-117.24(7)$ \\
\hline $\mathrm{O} 42 \mathrm{~B}-\mathrm{K} 3-\mathrm{O} 2 \mathrm{~W}-\mathrm{K} 2$ & $-131.64(8)$ \\
\hline $\mathrm{S} 4 \mathrm{~B}-\mathrm{K} 3-\mathrm{O} 41 \mathrm{~B}-\mathrm{K} 1^{\mathrm{viii}}$ & $153.3(2)$ \\
\hline $\mathrm{O} 2 \mathrm{~W}-\mathrm{K} 3-\mathrm{O} 41 \mathrm{~B}-\mathrm{S} 4 \mathrm{~B}$ & $-12.71(17)$ \\
\hline $\mathrm{O} 2 \mathrm{~W}-\mathrm{K} 3-\mathrm{O} 41 \mathrm{~B}-\mathrm{K} 1^{\mathrm{viii}}$ & $140.62(11)$ \\
\hline
\end{tabular}

\begin{tabular}{|c|c|}
\hline $\mathrm{O} 42 \mathrm{~A}-\mathrm{S} 4 \mathrm{~A}-\mathrm{O} 43 \mathrm{~A}-\mathrm{K} 3^{\mathrm{xii}}$ & $16.77(18)$ \\
\hline $\mathrm{O} 42 \mathrm{~A}-\mathrm{S} 4 \mathrm{~A}-\mathrm{O} 43 \mathrm{~A}-\mathrm{K} 1^{\mathrm{x}}$ & $113.45(15)$ \\
\hline $\mathrm{C} 4 \mathrm{~A}-\mathrm{S} 4 \mathrm{~A}-\mathrm{O} 43 \mathrm{~A}-\mathrm{K}^{\mathrm{xii}}$ & $131.69(15)$ \\
\hline $\mathrm{C} 4 \mathrm{~A}-\mathrm{S} 4 \mathrm{~A}-\mathrm{O} 43 \mathrm{~A}-\mathrm{K} 1^{\mathrm{x}}$ & $-131.62(15)$ \\
\hline $\mathrm{O} 41 \mathrm{~A}-\mathrm{S} 4 \mathrm{~A}-\mathrm{C} 4 \mathrm{~A}-\mathrm{C} 3 \mathrm{~A}$ & $59.0(4)$ \\
\hline $\mathrm{O} 41 \mathrm{~A}-\mathrm{S} 4 \mathrm{~A}-\mathrm{C} 4 \mathrm{~A}-\mathrm{C} 5 \mathrm{~A}$ & $-122.4(4)$ \\
\hline $\mathrm{O} 42 \mathrm{~A}-\mathrm{S} 4 \mathrm{~A}-\mathrm{C} 4 \mathrm{~A}-\mathrm{C} 3 \mathrm{~A}$ & $-60.9(4)$ \\
\hline $\mathrm{O} 42 \mathrm{~A}-\mathrm{S} 4 \mathrm{~A}-\mathrm{C} 4 \mathrm{~A}-\mathrm{C} 5 \mathrm{~A}$ & $117.7(4)$ \\
\hline $\mathrm{O} 43 \mathrm{~A}-\mathrm{S} 4 \mathrm{~A}-\mathrm{C} 4 \mathrm{~A}-\mathrm{C} 3 \mathrm{~A}$ & $179.8(3)$ \\
\hline $\mathrm{O} 43 \mathrm{~A}-\mathrm{S} 4 \mathrm{~A}-\mathrm{C} 4 \mathrm{~A}-\mathrm{C} 5 \mathrm{~A}$ & $-1.6(4)$ \\
\hline $\mathrm{K} 3-\mathrm{S} 4 \mathrm{~B}-\mathrm{O} 41 \mathrm{~B}-\mathrm{K} 1^{\mathrm{viii}}$ & $-144.8(3)$ \\
\hline $\mathrm{O} 42 \mathrm{~B}-\mathrm{S} 4 \mathrm{~B}-\mathrm{O} 41 \mathrm{~B}-\mathrm{K} 3$ & $-5.2(2)$ \\
\hline $\mathrm{O} 42 \mathrm{~B}-\mathrm{S} 4 \mathrm{~B}-\mathrm{O} 41 \mathrm{~B}-\mathrm{K} 1^{\mathrm{viii}}$ & $-150.0(2)$ \\
\hline $\mathrm{O} 43 \mathrm{~B}-\mathrm{S} 4 \mathrm{~B}-\mathrm{O} 41 \mathrm{~B}-\mathrm{K} 3$ & $122.13(18)$ \\
\hline $\mathrm{O} 43 \mathrm{~B}-\mathrm{S} 4 \mathrm{~B}-\mathrm{O} 41 \mathrm{~B}-\mathrm{K} 1^{\mathrm{viii}}$ & $-22.7(3)$ \\
\hline $\mathrm{C} 4 \mathrm{~B}-\mathrm{S} 4 \mathrm{~B}-\mathrm{O} 41 \mathrm{~B}-\mathrm{K} 3$ & $-119.08(16)$ \\
\hline $\mathrm{C} 4 \mathrm{~B}-\mathrm{S} 4 \mathrm{~B}-\mathrm{O} 41 \mathrm{~B}-\mathrm{K} 1^{\text {viii }}$ & $96.1(2)$ \\
\hline $\mathrm{O} 41 \mathrm{~B}-\mathrm{S} 4 \mathrm{~B}-\mathrm{O} 42 \mathrm{~B}-\mathrm{K} 3$ & $4.44(17)$ \\
\hline $\mathrm{O} 43 \mathrm{~B}-\mathrm{S} 4 \mathrm{~B}-\mathrm{O} 42 \mathrm{~B}-\mathrm{K} 3$ & $-124.95(15)$ \\
\hline $\mathrm{C} 4 \mathrm{~B}-\mathrm{S} 4 \mathrm{~B}-\mathrm{O} 42 \mathrm{~B}-\mathrm{K} 3$ & $118.76(15)$ \\
\hline $\mathrm{K} 3-\mathrm{S} 4 \mathrm{~B}-\mathrm{O} 43 \mathrm{~B}-\mathrm{K} 1$ & $-44.0(2)$ \\
\hline $\mathrm{K} 3-\mathrm{S} 4 \mathrm{~B}-\mathrm{O} 43 \mathrm{~B}-\mathrm{K} 1^{\mathrm{xiii}}$ & $60.7(3)$ \\
\hline $\mathrm{O} 41 \mathrm{~B}-\mathrm{S} 4 \mathrm{~B}-\mathrm{O} 43 \mathrm{~B}-\mathrm{K} 1$ & $-100.68(18)$ \\
\hline $\mathrm{O} 41 \mathrm{~B}-\mathrm{S} 4 \mathrm{~B}-\mathrm{O} 43 \mathrm{~B}-\mathrm{K} 1^{\mathrm{xiii}}$ & $4.0(3)$ \\
\hline $\mathrm{O} 42 \mathrm{~B}-\mathrm{S} 4 \mathrm{~B}-\mathrm{O} 43 \mathrm{~B}-\mathrm{K} 1$ & $26.9(2)$ \\
\hline $\mathrm{O} 42 \mathrm{~B}-\mathrm{S} 4 \mathrm{~B}-\mathrm{O} 43 \mathrm{~B}-\mathrm{K} 1^{\mathrm{xiii}}$ & $131.6(3)$ \\
\hline $\mathrm{C} 4 \mathrm{~B}-\mathrm{S} 4 \mathrm{~B}-\mathrm{O} 43 \mathrm{~B}-\mathrm{K} 1$ & $141.61(16)$ \\
\hline $\mathrm{C} 4 \mathrm{~B}-\mathrm{S} 4 \mathrm{~B}-\mathrm{O} 43 \mathrm{~B}-\mathrm{K} 1^{\mathrm{xiii}}$ & $-113.7(3)$ \\
\hline $\mathrm{K} 3-\mathrm{S} 4 \mathrm{~B}-\mathrm{C} 4 \mathrm{~B}-\mathrm{C} 3 \mathrm{~B}$ & $142.0(3)$ \\
\hline $\mathrm{K} 3-\mathrm{S} 4 \mathrm{~B}-\mathrm{C} 4 \mathrm{~B}-\mathrm{C} 5 \mathrm{~B}$ & $-36.5(5)$ \\
\hline $\mathrm{O} 41 \mathrm{~B}-\mathrm{S} 4 \mathrm{~B}-\mathrm{C} 4 \mathrm{~B}-\mathrm{C} 3 \mathrm{~B}$ & $-166.4(3)$ \\
\hline $\mathrm{O} 41 \mathrm{~B}-\mathrm{S} 4 \mathrm{~B}-\mathrm{C} 4 \mathrm{~B}-\mathrm{C} 5 \mathrm{~B}$ & $15.1(5)$ \\
\hline $\mathrm{O} 42 \mathrm{~B}-\mathrm{S} 4 \mathrm{~B}-\mathrm{C} 4 \mathrm{~B}-\mathrm{C} 3 \mathrm{~B}$ & $75.4(4)$ \\
\hline $\mathrm{O} 42 \mathrm{~B}-\mathrm{S} 4 \mathrm{~B}-\mathrm{C} 4 \mathrm{~B}-\mathrm{C} 5 \mathrm{~B}$ & $-103.1(5)$ \\
\hline $\mathrm{O} 43 \mathrm{~B}-\mathrm{S} 4 \mathrm{~B}-\mathrm{C} 4 \mathrm{~B}-\mathrm{C} 3 \mathrm{~B}$ & $-43.0(4)$ \\
\hline $\mathrm{O} 43 \mathrm{~B}-\mathrm{S} 4 \mathrm{~B}-\mathrm{C} 4 \mathrm{~B}-\mathrm{C} 5 \mathrm{~B}$ & $138.5(4)$ \\
\hline $\mathrm{C} 2 \mathrm{~A}-\mathrm{C} 1 \mathrm{~A}-\mathrm{C} 1 \mathrm{~B}-\mathrm{C} 2 \mathrm{~B}$ & $3.0(6)$ \\
\hline $\mathrm{C} 2 \mathrm{~A}-\mathrm{C} 1 \mathrm{~A}-\mathrm{C} 1 \mathrm{~B}-\mathrm{C} 6 \mathrm{~B}$ & $-178.3(5)$ \\
\hline $\mathrm{C} 6 \mathrm{~A}-\mathrm{C} 1 \mathrm{~A}-\mathrm{C} 1 \mathrm{~B}-\mathrm{C} 2 \mathrm{~B}$ & $-176.3(4)$ \\
\hline $\mathrm{C} 6 \mathrm{~A}-\mathrm{C} 1 \mathrm{~A}-\mathrm{C} 1 \mathrm{~B}-\mathrm{C} 6 \mathrm{~B}$ & $2.5(7)$ \\
\hline $\mathrm{C} 1 \mathrm{~B}-\mathrm{C} 1 \mathrm{~A}-\mathrm{C} 2 \mathrm{~A}-\mathrm{C} 3 \mathrm{~A}$ & $-177.7(4)$ \\
\hline $\mathrm{C} 6 \mathrm{~A}-\mathrm{C} 1 \mathrm{~A}-\mathrm{C} 2 \mathrm{~A}-\mathrm{C} 3 \mathrm{~A}$ & $1.6(7)$ \\
\hline
\end{tabular}




\section{supplementary materials}

\begin{tabular}{|c|c|c|c|}
\hline $\mathrm{O} 42 \mathrm{~B}-\mathrm{K} 3-\mathrm{O} 41 \mathrm{~B}-\mathrm{S} 4 \mathrm{~B}$ & $3.06(12)$ & $\mathrm{C} 1 \mathrm{~B}-\mathrm{C} 1 \mathrm{~A}-\mathrm{C} 6 \mathrm{~A}-\mathrm{C} 5 \mathrm{~A}$ & $178.1(5)$ \\
\hline $\mathrm{O} 42 \mathrm{~B}-\mathrm{K} 3-\mathrm{O} 41 \mathrm{~B}-\mathrm{K} 1^{\mathrm{viii}}$ & $156.39(16)$ & $\mathrm{C} 2 \mathrm{~A}-\mathrm{C} 1 \mathrm{~A}-\mathrm{C} 6 \mathrm{~A}-\mathrm{C} 5 \mathrm{~A}$ & $-1.2(8)$ \\
\hline $\mathrm{O} 42 \mathrm{~A}^{\mathrm{iii}}-\mathrm{K} 3-\mathrm{O} 41 \mathrm{~B}-\mathrm{S} 4 \mathrm{~B}$ & $-75.87(15)$ & $\mathrm{C} 1 \mathrm{~A}-\mathrm{C} 1 \mathrm{~B}-\mathrm{C} 2 \mathrm{~B}-\mathrm{C} 3 \mathrm{~B}$ & $178.6(4)$ \\
\hline $\mathrm{O} 43 \mathrm{~A}^{\mathrm{iii}}-\mathrm{K} 3-\mathrm{O} 41 \mathrm{~B}-\mathrm{S} 4 \mathrm{~B}$ & $-125.62(17)$ & $\mathrm{C} 6 \mathrm{~B}-\mathrm{C} 1 \mathrm{~B}-\mathrm{C} 2 \mathrm{~B}-\mathrm{C} 3 \mathrm{~B}$ & $-0.2(7)$ \\
\hline $\mathrm{O} 2 \mathrm{~W}^{\mathrm{viii}}-\mathrm{K} 3-\mathrm{O} 41 \mathrm{~B}-\mathrm{S} 4 \mathrm{~B}$ & $154.69(18)$ & $\mathrm{C} 1 \mathrm{~A}-\mathrm{C} 1 \mathrm{~B}-\mathrm{C} 6 \mathrm{~B}-\mathrm{C} 5 \mathrm{~B}$ & $-178.0(6)$ \\
\hline$S 4 B^{i x}-K 3-O 41 B-S 4 B$ & $70.76(16)$ & $\mathrm{C} 2 \mathrm{~B}-\mathrm{C} 1 \mathrm{~B}-\mathrm{C} 6 \mathrm{~B}-\mathrm{C} 5 \mathrm{~B}$ & $0.8(9)$ \\
\hline $\mathrm{O} 41 \mathrm{~B}^{\mathrm{ix}}-\mathrm{K} 3-\mathrm{O} 41 \mathrm{~B}-\mathrm{S} 4 \mathrm{~B}$ & $98.52(16)$ & $\mathrm{C} 1 \mathrm{~A}-\mathrm{C} 2 \mathrm{~A}-\mathrm{C} 3 \mathrm{~A}-\mathrm{C} 4 \mathrm{~A}$ & $-0.5(7)$ \\
\hline $\mathrm{O} 42 \mathrm{~B}^{\mathrm{ix}}-\mathrm{K} 3-\mathrm{O} 41 \mathrm{~B}-\mathrm{S} 4 \mathrm{~B}$ & $43.89(18)$ & $\mathrm{C} 1 \mathrm{~B}-\mathrm{C} 2 \mathrm{~B}-\mathrm{C} 3 \mathrm{~B}-\mathrm{C} 4 \mathrm{~B}$ & $-1.5(6)$ \\
\hline 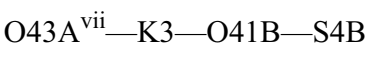 & $-165.15(13)$ & $\mathrm{C} 2 \mathrm{~A}-\mathrm{C} 3 \mathrm{~A}-\mathrm{C} 4 \mathrm{~A}-\mathrm{S} 4 \mathrm{~A}$ & $177.5(4)$ \\
\hline $\mathrm{O} 2 \mathrm{~W}-\mathrm{K} 3-\mathrm{O} 42 \mathrm{~B}-\mathrm{S} 4 \mathrm{~B}$ & $158.11(18)$ & $\mathrm{C} 2 \mathrm{~A}-\mathrm{C} 3 \mathrm{~A}-\mathrm{C} 4 \mathrm{~A}-\mathrm{C} 5 \mathrm{~A}$ & $-1.1(7)$ \\
\hline $\mathrm{O} 41 \mathrm{~B}-\mathrm{K} 3-\mathrm{O} 42 \mathrm{~B}-\mathrm{S} 4 \mathrm{~B}$ & $-2.91(11)$ & $\mathrm{C} 2 \mathrm{~B}-\mathrm{C} 3 \mathrm{~B}-\mathrm{C} 4 \mathrm{~B}-\mathrm{S} 4 \mathrm{~B}$ & $-175.8(3)$ \\
\hline $\mathrm{O} 42 \mathrm{~A}^{\mathrm{iii}}-\mathrm{K} 3-\mathrm{O} 42 \mathrm{~B}-\mathrm{S} 4 \mathrm{~B}$ & $83.00(14)$ & $\mathrm{C} 2 \mathrm{~B}-\mathrm{C} 3 \mathrm{~B}-\mathrm{C} 4 \mathrm{~B}-\mathrm{C} 5 \mathrm{~B}$ & $2.7(7)$ \\
\hline $\mathrm{O} 43 \mathrm{~A}^{\mathrm{iii}}-\mathrm{K} 3-\mathrm{O} 42 \mathrm{~B}-\mathrm{S} 4 \mathrm{~B}$ & $46.38(15)$ & $\mathrm{S} 4 \mathrm{~A}-\mathrm{C} 4 \mathrm{~A}-\mathrm{C} 5 \mathrm{~A}-\mathrm{C} 6 \mathrm{~A}$ & $-177.1(4)$ \\
\hline $\mathrm{O} 2 \mathrm{~W}^{\mathrm{viii}}-\mathrm{K} 3-\mathrm{O} 42 \mathrm{~B}-\mathrm{S} 4 \mathrm{~B}$ & $-33.08(17)$ & $\mathrm{C} 3 \mathrm{~A}-\mathrm{C} 4 \mathrm{~A}-\mathrm{C} 5 \mathrm{~A}-\mathrm{C} 6 \mathrm{~A}$ & $1.5(7)$ \\
\hline $\mathrm{S} 4 \mathrm{~B}^{\mathrm{ix}}-\mathrm{K} 3-\mathrm{O} 42 \mathrm{~B}-\mathrm{S} 4 \mathrm{~B}$ & $-130.57(13)$ & $\mathrm{S} 4 \mathrm{~B}-\mathrm{C} 4 \mathrm{~B}-\mathrm{C} 5 \mathrm{~B}-\mathrm{C} 6 \mathrm{~B}$ & $176.4(5)$ \\
\hline $\mathrm{O} 41 \mathrm{~B}^{\mathrm{ix}}-\mathrm{K} 3-\mathrm{O} 42 \mathrm{~B}-\mathrm{S} 4 \mathrm{~B}$ & $-114.34(14)$ & $\mathrm{C} 3 \mathrm{~B}-\mathrm{C} 4 \mathrm{~B}-\mathrm{C} 5 \mathrm{~B}-\mathrm{C} 6 \mathrm{~B}$ & $-2.1(9)$ \\
\hline $\mathrm{O} 42 \mathrm{~B}^{\mathrm{ix}}-\mathrm{K} 3-\mathrm{O} 42 \mathrm{~B}-\mathrm{S} 4 \mathrm{~B}$ & $-144.75(16)$ & $\mathrm{C} 4 \mathrm{~A}-\mathrm{C} 5 \mathrm{~A}-\mathrm{C} 6 \mathrm{~A}-\mathrm{C} 1 \mathrm{~A}$ & $-0.3(8)$ \\
\hline 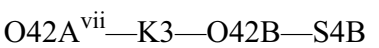 & $158.31(12)$ & $\mathrm{C} 4 \mathrm{~B}-\mathrm{C} 5 \mathrm{~B}-\mathrm{C} 6 \mathrm{~B}-\mathrm{C} 1 \mathrm{~B}$ & $0.4(10)$ \\
\hline
\end{tabular}

Symmetry codes: (i) $x-1, y, z$; (ii) $x-1, y, z+1$; (iii) $x, y, z+1$; (iv) $x-1 / 2,-y+1 / 2, z$; (v) $x-1 / 2,-y+1 / 2, z+1$; (vi) $x-1,-y, z+1$; (vii) $x,-y$, $z+1$; (viii) $x+1, y, z$; (ix) $x,-y, z$; (x) $x+1, y, z-1$; (xi) $x+1 / 2,-y+1 / 2, z-1$; (xii) $x, y, z-1$; (xiii) $x+1 / 2,-y+1 / 2, z$.

Hydrogen-bond geometry $\left(A,{ }^{\circ}\right)$

\begin{tabular}{|c|c|c|c|c|}
\hline$D-\mathrm{H} \cdots A$ & $D-\mathrm{H}$ & $\mathrm{H} \cdots A$ & $D \cdots A$ & $D-\mathrm{H} \cdots A$ \\
\hline $\mathrm{O} 1 \mathrm{~W}-\mathrm{H} 11 \mathrm{~W} \cdots \mathrm{O} 4 \mathrm{~W}^{\mathrm{iii}}$ & 0.97 & 2.18 & $2.778(9)$ & 119 \\
\hline $\mathrm{O} 2 \mathrm{~W}-\mathrm{H} 2 \mathrm{~W} \cdots \mathrm{O} 42 \mathrm{~B}$ & 0.96 & 1.91 & $2.840(5)$ & 162 \\
\hline $\mathrm{O} 3 \mathrm{~W}-\mathrm{H} 3 \mathrm{~W} \cdots \mathrm{O} 42 \mathrm{~B}$ & 0.96 & 1.95 & $2.884(5)$ & 162 \\
\hline $\mathrm{O} 4 \mathrm{~W}-\mathrm{H} 41 \mathrm{~W} \cdots \mathrm{O} 3 \mathrm{~W}$ & 0.90 & 1.82 & $2.715(9)$ & 180 \\
\hline $\mathrm{O} 4 \mathrm{~W}-\mathrm{H} 41 \mathrm{~W} \cdots \mathrm{O} 3 \mathrm{~W}$ & 0.90 & 1.82 & $2.715(9)$ & 180 \\
\hline $\mathrm{C} 5 \mathrm{~A}-\mathrm{H} 5 \mathrm{~A} \cdots \mathrm{O} 43 \mathrm{~A}$ & 0.95 & 2.50 & $2.916(5)$ & 106 \\
\hline $\mathrm{C} 5 \mathrm{~B}-\mathrm{H} 5 \mathrm{~B} \cdots \mathrm{O} 41 \mathrm{~B}$ & 0.94 & 2.47 & $2.873(5)$ & 106 \\
\hline
\end{tabular}

Symmetry codes: (iii) $x, y, z+1$. 
Fig. 1

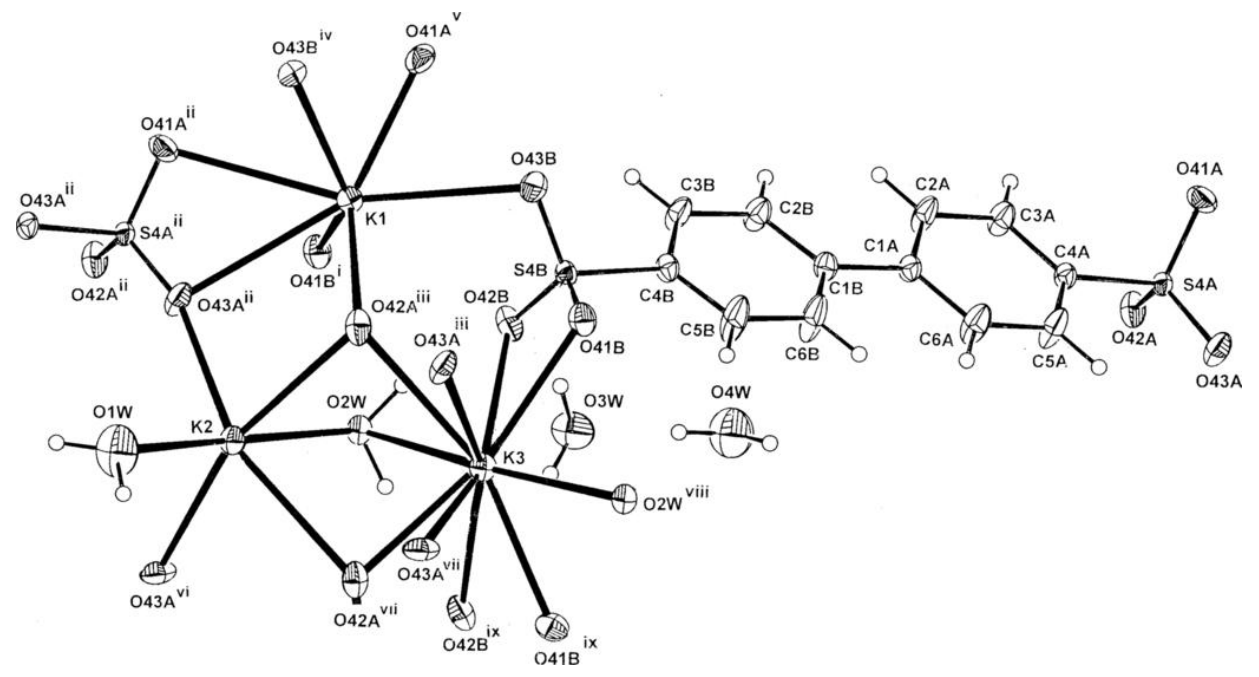




\section{supplementary materials}

Fig. 2

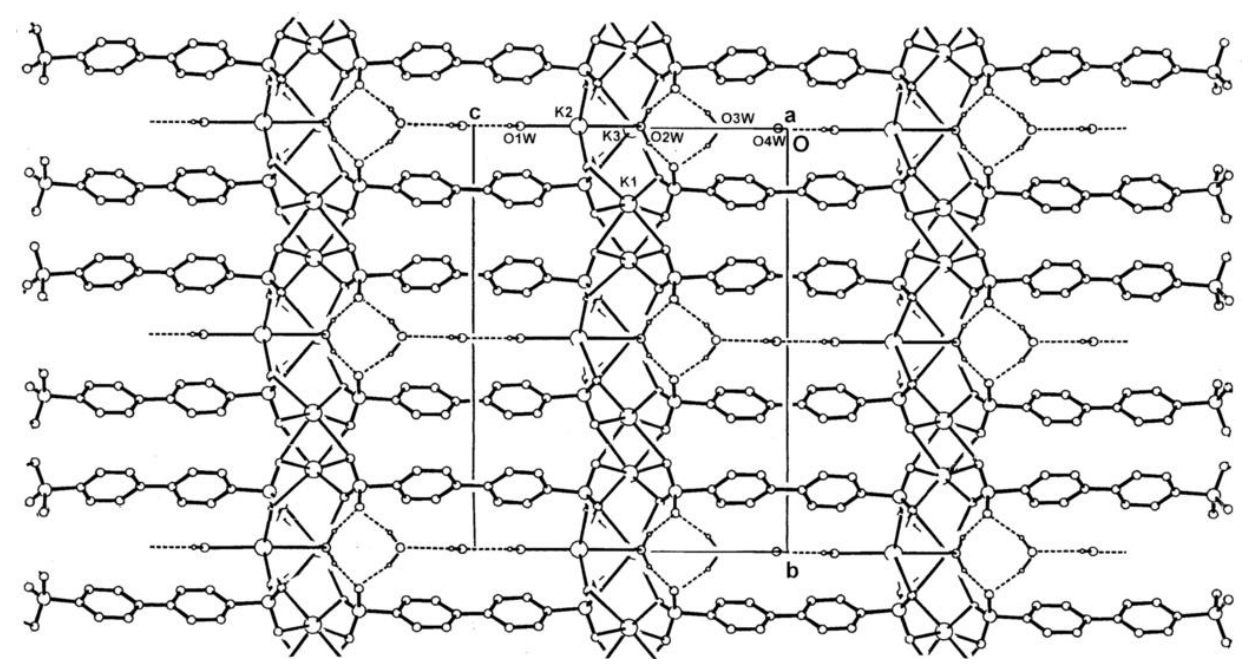

